\title{
Photochemical and Electrochemical Strategies towards Benzylic C-H Functionalization: A Recent Update
}

\author{
Monica Oliva, ${ }^{+a}$ Guglielmo A. Coppola, ${ }^{+a}$ Erik V. Van der Eycken,,${ }^{\mathrm{a} b}$ and \\ Upendra K. Sharma ${ }^{\mathrm{a}, *}$ \\ a Laboratory for Organic \& Microwave-Assisted Chemistry (LOMAC), Department of Chemistry, \\ University of Leuven (KU Leuven), Celestijnenlaan 200F, B-3001, Leuven, Belgium \\ E-mail: upendrakumar.sharma@kuleuven.be; usharma81@gmail.com \\ b Peoples' Friendship University of Russia (RUDN University), \\ 6 Miklukho-Maklaya street, RU-117198, Moscow, Russia
}

These authors contributed equally and share first authorship.

Manuscript received: December 20, 2020; Revised manuscript received: February 3, 2021;

Version of record online:

Abstract: Transition metal-catalysed processes have been widely used for the functionalization of inert $\mathrm{C}-\mathrm{H}$ bonds. Strategies for the functionalization of the benzylic $\mathrm{C}-\mathrm{H}$ position having a relatively weak $\mathrm{C}-\mathrm{H}$ bond (bond dissociation energy 80-90 kcal $/ \mathrm{mol}$ ) differ from the inert aliphatic and aromatic $\mathrm{C}-\mathrm{H}$ positions with stronger $\mathrm{C}-\mathrm{H}$ bonds. The recent advances in the direct activation of the benzylic position through the generation of $\mathrm{C}\left(s p^{3}\right)$ radicals have demonstrated the potential of electrochemistry and photochemistry as a means for constructing new chemical bonds. This review will cover the recent progress of benzylic $\mathrm{C}-\mathrm{H}$ functionalization through organic radical strategies employing photochemistry and electrochemistry as sustainable tools. In addition, the mechanistic details of the typical reactions have been included which, in turn, will help the researchers to look at this promising area from a different perspective towards new discoveries and often hidden opportunities.

\section{Introduction}

2. Photo-Induced Benzylic C-H Functionalization

2.1. $\mathrm{C}\left(s p^{3}\right)-\mathrm{C}$ Bond Formation

2.2. $\mathrm{C}\left(s p^{2}\right)-\mathrm{O}$ and $\mathrm{C}\left(s p^{3}\right)-\mathrm{O}$ Bond Formation

2.3. $\mathrm{C}\left(s p^{3}\right)-\mathrm{N}$ Bond Formation

2.4. $\mathrm{C}\left(s p^{3}\right)-\mathrm{S}$ Bond Formation

2.5. $\mathrm{C}\left(s p^{3}\right)-$ Halogen Bond Formation

3. Electrochemical Benzylic $\mathrm{C}\left(s p^{3}\right)-\mathrm{H}$ Functionalization

3.1. $\mathrm{C}\left(s p^{3}\right)-\mathrm{C}$ Bond Formation

3.2. $\mathrm{C}\left(s p^{3}\right)-\mathrm{O}$ Bond Formation

3.3. $\mathrm{C}\left(s p^{3}\right)-\mathrm{N}$ Bond Formation

3.4. $\mathrm{C}\left(s p^{3}\right)-\mathrm{P}$ Bond Formation

3.5. $\mathrm{C}\left(s p^{3}\right)$-Halogen Bond Formation

4. Benzylic $\mathrm{C}\left(s p^{3}\right)-\mathrm{H}$ Functionalization via Synthetic Photoelectrochemistry

5. Conclusions and Outlook

Keywords: benzylic $\mathrm{C}-\mathrm{H}$ functionalization; photochemistry; electrochemistry; photoelectrochemistry; photocatalysis

\section{Introduction}

Among the various $\mathrm{C}-\mathrm{H}$ bonds, direct functionalization of the benzylic position is particularly fascinating and at the same time a challenging process for synthetic chemists; moreover, alkyl arenes are ubiquitous and their selective functionalization can have tremendous impact in feedstock and fine chemistry. Since these hydrocarbons are abundant and usually cheap petroleum derivatives, their use as building blocks has an obvious economic appeal for the synthesis of valuable compounds without the need of pre-functionalization. Furthermore, late stage decoration of complex molecules and pharmaceuticals can greatly benefit from strategies that allow for direct access to the benzylic position. ${ }^{[1, b]}$

In the context of $\mathrm{C}-\mathrm{H}$ functionalization, chemoand regioselectivity represent the major challenges. Therefore, reactivity differences have to be evaluated in order to successfully achieve selective functionalization and the activation strategy needs to be tailored to the $\mathrm{C}-\mathrm{H}$ bond we aim to activate. ${ }^{[\mathrm{lc}]}$ Luckily, nowadays a plethora of different strategies are available, and 


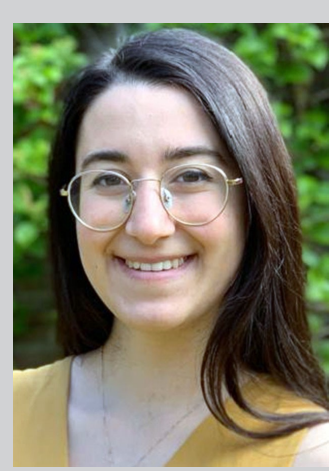

Monica Oliva received her Master's degree in pharmaceutical chemistry and technology in 2018 at the University of Siena. Later, she joined LOMAC as an Erasmus ${ }^{+}$trainee working on odourless isocyanide chemistry towards transition metal-catalysis. She is currently pursuing her PhD under the supervision of Prof. Dr. Erik Van der Eycken and Dr. Upendra K. Sharma at KU Leuven.

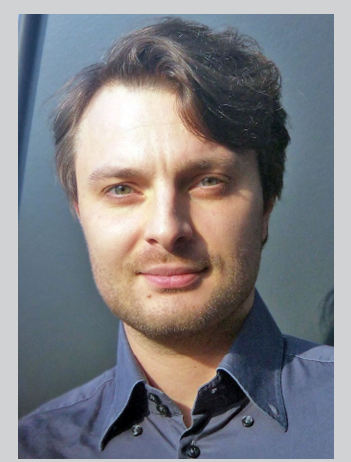

Guglielmo A. Coppola received his Master's degree in pharmaceutical chemistry and technology (2017) from Sapienza University of Rome. Later in the same year he joined LOMAC in the frame of an Erasmus ${ }^{+}$ scholarship working on metalfree spirocyclizations. He is currently working as a PhDstudent under the supervision of Prof. Erik Van der Eycken and Prof. Hans Steenackers at KU Leuven. His research focuses on the synthesis of bioactive heterocycles.

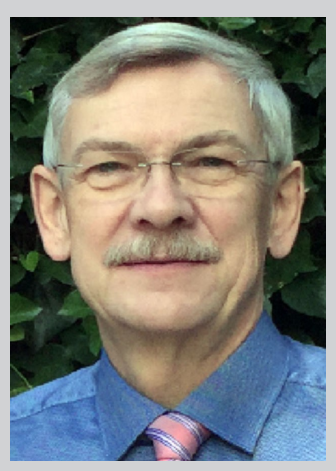

Erik V. Van der Eycken is Full Professor of Organic Chemistry and Head of the Division of Molecular Design \& Synthesis at KU Leuven, Belgium. He received his $P h D$ degree (1987) in organic chemistry from the University of Ghent, Belgium. He spent time as visiting scientist at the University of Graz (2002) with Prof. C. O. Kappe, at The Scripps Research Institute (La Jolla, USA) (2003) in the group of K. B. Sharpless, and at Uppsala University (2004) with Prof. M. Larhed and Prof. A. Hallberg. The main focus of his research is the development of new synthetic methodologies in combination with enabling techniques.

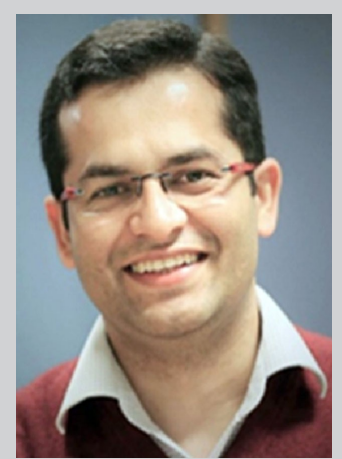

Upendra K. Sharma received his PhD (2011) from CSIRInstitute of Himalayan Bioresource Technology, Palampur, India. Thereafter, he worked as an assistant professor for a short period at the National Institute of Technology, Jalandhar, India. In 2013, he joined the research group of Prof. Erik Van der Eycken at the University of Leuven, Belgium followed by postdoctoral stints with Prof. Steven Ley (University of Cambridge), Prof. Timothy Noël (University of Eindhoven) and Prof. Shu-Li You (SIOC, China). In 2020, he joined KU Leuven as a senior research-expert. His research interests include new reaction methodologies, photoredox catalysis, $\mathrm{C}-\mathrm{H}$ functionalizations and flow chemistry. careful evaluation of the electronic and steric environment around the $\mathrm{C}-\mathrm{H}$ bond to be reacted, can allow for the installation of functional groups with surgical precision. ${ }^{[2]}$ Transition metal promoted $\mathrm{C}-\mathrm{H}$ activation and subsequent functionalization has gained much success in the last decade, especially for $\mathrm{C}\left(s p^{2}\right)-\mathrm{H}$ bonds. ${ }^{[3]}$ Nonetheless, lower applicability to $\mathrm{C}\left(s p^{3}\right)-\mathrm{H}$ functionalization has restrained its scope. ${ }^{[4]}$ In an attempt to explain this trend, we could analyse the correlation between bond dissociation energies (BDEs) of $\mathrm{C}-\mathrm{H}$ bonds with different hybridization, just to discover that $\mathrm{C}\left(s p^{3}\right)-\mathrm{H}$ appear to be the weakest. Siegbahn, ${ }^{[5]}$ on the basis of Jones's and Feher's research, ${ }^{[6]}$ attributed this fact to carbon-metal bond energies, which follow an opposite trend, to determine whether the activation will be successful or not. With these data in hand, a straightforward solution would be to stop only relying on transition-metal activation, and instead focusing on processes that would rather be mainly regulated by BDEs. This is the case for radical processes where homolytic dissociation of $\mathrm{C}-\mathrm{H}$ bonds generates carbon centred radicals, which are able to react with the opportune counterpart towards the formation of $\mathrm{C}-\mathrm{C}$ bonds as well as $\mathrm{C}$-heteroatom bonds. In addition, greater attention on availability, cost, and toxicity of heavier transition metal catalysts like $\mathrm{Pd}, \mathrm{Pt}$ and $\mathrm{Rh}$ has shifted the focus to more abundant, cheaper, and less toxic ones such as copper and iron, paving the way for the development of radical cross-coupling reactions. ${ }^{[7]}$ Nonetheless, these examples of radical chemistry rely on the use of both stoichiometric oxidants and high temperatures, which in turn, can become major reasons to withdraw from these strategies due to economic, safety and environ- 
mental issues. In the past decades, synthetic chemists have ridden the wave of green chemistry which set major challenges resulting, thereafter, in tremendous improvements and innovation. Photoredox catalysis and electrochemistry have shone new light on radical chemistry, allowing organic chemists to harness free radicals by fine tuning the redox behaviour of the substrates towards higher selectivity and applicability. Moreover, since the activation of the substrates involves mainly single electron transfer or energy transfer, these reactions are usually carried at room temperature contrarily to thermal processes. Direct functionalization of benzylic $\mathrm{C}-\mathrm{H}$ bonds has been thoroughly investigated being, among the $\mathrm{C}\left(s p^{3}\right)-\mathrm{H}$ sites, the most accessible. ${ }^{[6]}$ In this review we will provide an overview of the recent progresses in photo/ electro- or photoelectron-catalysed processes for the direct functionalization of the benzylic $\mathrm{C}-\mathrm{H}$ bond.

\section{Photo-Induced Benzylic $\mathrm{C}-\mathrm{H}$ Functionalization}

Intensive research in the field of visible-light photoredox catalysis has offered new ways to easily access radical processes in a more selective manner. Moreover, the development of metal-centred photocatalysts as well as organic dyes, together with an easy access to reliable light sources has allowed for a rapid acceleration of this field. Photocatalysis has offered sustainable alternatives to more conventional processes, shifting the attention to the reactivity of the radical intermediates as well as their precursor, thus giving access to novel synthetic designs. In this section, we will review the latest progress in the field of photoinduced $\mathrm{C}-\mathrm{H}$ functionalization, where a benzylic carbon-centred radical is formed either by single electron transfer (SET) or by direct hydrogen atom transfer (HAT).

The latter is a particularly intriguing example of an innate $\mathrm{C}-\mathrm{H}$ activation approach. As shown schematically in Scheme 1, direct abstraction of a hydrogen atom generates a carbon-centred radical intermediate able to undergo further functionalization.

The main factor influencing the reactivity is the inherent BDE of the $\mathrm{C}-\mathrm{H}$ bond.$^{[8-9]} \mathrm{HAT}$ represents a key step in a wide variety of chemical reactions and provides a unique opportunity in organic synthesis to allow the straightforward activation of $\mathrm{C}-\mathrm{H}$ bonds, often in a highly selective fashion. ${ }^{[10]}$ Recently, photo-

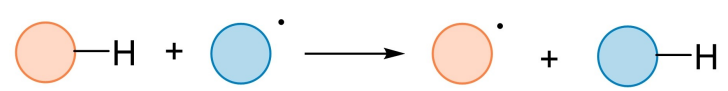

Scheme 1. Schematic representation of hydrogen atom transfer (HAT). catalysis has contributed significantly to the development of the field leading to a tremendous expansion of HAT-methodologies. ${ }^{[11]}$ Several classes of compounds have been found as suitable HAT reagents such as aryl ketones, ${ }^{[12]}$ polyoxometalates, ${ }^{[13]}$ thiols ${ }^{[14]}$ and other oxygen- and sulfur-based abstractors. ${ }^{[15]}$ Proper combination of a photocatalytic system and a hydrogen abstractor can lead to the selective cleavage of $\mathrm{C}-\mathrm{H}$ bonds and subsequent functionalization.

\subsection{C $\left(s p^{3}\right)-C$ Bond Formation}

Pioneering work of MacMillan, Doyle and collaborators $^{[16]}$ towards achieving the challenging $\mathrm{C}$ $\left(s p^{3}\right)-\mathrm{C}\left(s p^{2}\right)$ bond formation, led to the realization of merging photoredox with nickel catalysis employing $\alpha$ amino acids as radical precursors. This finding marked the beginning of years of active research and improvements in the field to access new synthetic pathways in order to turn on reactivity on sites that would have otherwise remain unreactive. Molander and collaborators, ${ }^{[17]}$ riding the wave of dual catalysis, overcame the need of substrate pre-functionalization by achieving carbon-centred radicals using diaryl ketone as HAT reagents. The ability of aromatic ketones to abstract a hydrogen atom upon irradiation has been exploited extensively in photochemistry. ${ }^{[18]}$

This has been inspirational for several papers thereafter and led Zhan's group to develop an efficient protocol for the enantioselective benzylic $\mathrm{C}\left(s p^{3}\right)-\mathrm{H}$ bond arylation via photoredox/nickel dual catalysis (Scheme 2A). ${ }^{[19]}$ The scarce availability of ligands that are able to differentiate between competing diastereomeric transition states makes asymmetric cross-coupling reaction via this photocatalytic HAT process quite challenging. To overcome this, Zhan's group successfully synthesized asymmetric 1,1-diaryl alkanes using in-house designed chiral biimidazoline ligand L1 (Scheme 2B) and dimethyl benzophenone (DMBP) as HAT reagent. However, DMBP acted mainly as a

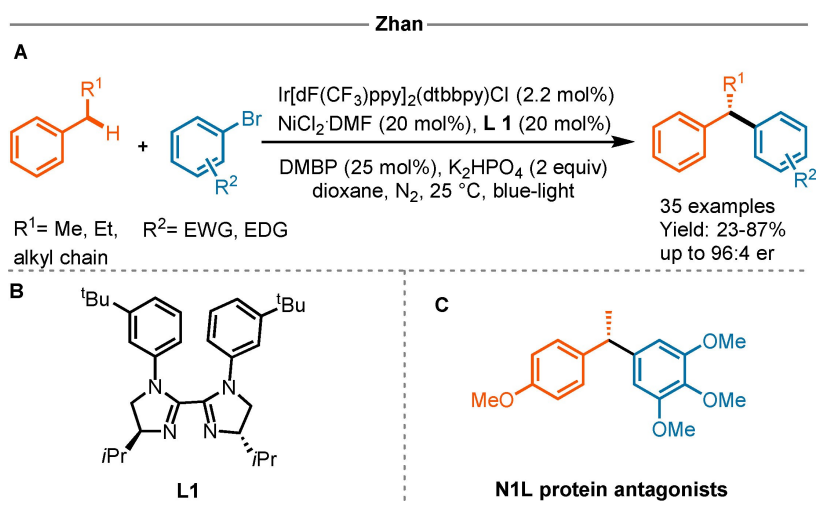

Scheme 2. Enantioselective benzylic $\mathrm{C}-\mathrm{H}$ arylation via photoredox and nickel dual catalysis. 
cocatalyst as the hydrogen atom abstraction was carried out by the bromine-free radical generated from the nickel-aryl adduct. The photocatalytic cycle kept the protocol redox neutral overcoming the need of sacrificial redox reagents. The reaction conditions were successfully applied on a broad variety of aryl coupling partners generating products with good enantioselectivity, including complex structures and bioactive compounds (Scheme 2C).

Very recently, Murakami's group has reported another example of bromine radical promoted HAT. ${ }^{[20]}$ They performed a dehydrogenative $\mathrm{C}-\mathrm{H} / \mathrm{C}-\mathrm{H}$ crosscoupling reaction of alkylbenzenes with aldehydes to form $\alpha$-aryl ketones, promoted by iridium and nickel dual catalysis under blue-light irradiation (Scheme 3). Whereas electron-donating groups such as tert-butyl and siloxy groups were eligible substituents on the benzene ring, electron-withdrawing substituents such as alkoxycarbonyl, acyl, and cyano groups gave no cross-coupling products.

Since the HAT preferably occurs at electron rich benzylic positions, the authors suggested the attack from the bromine radical to occur in an electrophilic fashion. Mechanistic studies supported the oxidation of a bromine anion by the $\mathrm{Ni}$ catalyst to generate a radical. When $\operatorname{Ir}\left[\mathrm{dF}\left(\mathrm{CF}_{3}\right) \text { ppy }\right]_{2}(\mathrm{dtbbpy}) \mathrm{PF}_{6}$ was treated with $\mathrm{NiBr}_{2}(\mathrm{dtbbpy})$ in $\mathrm{CDCl}_{3}$, the hexafluorophosphate anion was replaced with a bromo ligand to form $\operatorname{Ir}\left[\mathrm{dF}\left(\mathrm{CF}_{3}\right) \text { ppy }\right]_{2}(\mathrm{dtbbpy}) \mathrm{Br}$. Consequently, photo-irradiation of $\operatorname{Ir}\left[\mathrm{dF}_{2}\left(\mathrm{CF}_{3}\right) \text { ppy }\right]_{2}(\mathrm{dtbbpy}) \mathrm{Br}$ induces a single electron transfer from the bromide anion to iridium, the resulting bromine radical abstracts hydrogen from aldehydes and alkanes.

Intrigued by the possibility of using simple halogen radicals as catalyst to abstract $\mathrm{H}$ atoms, $\mathrm{Wu}$ and

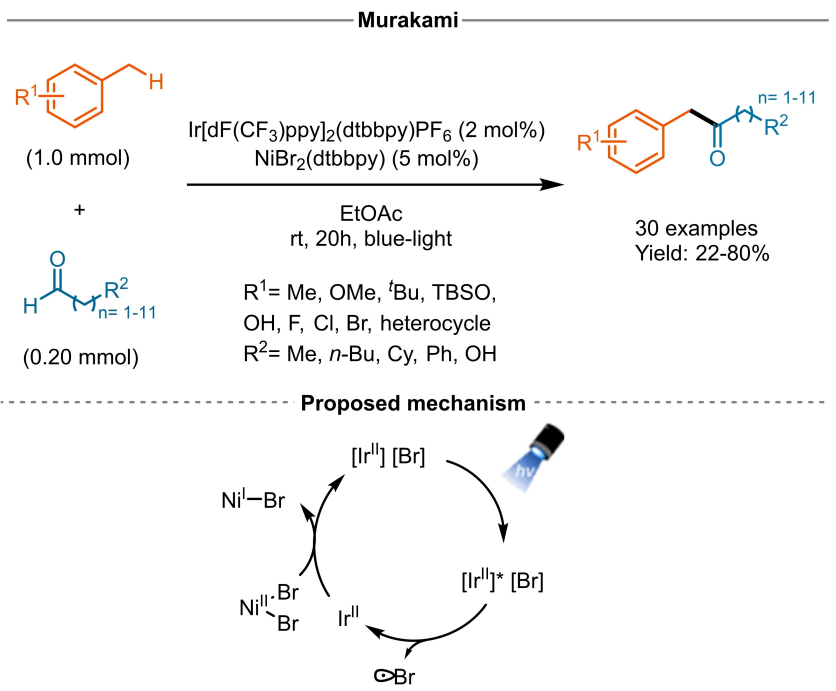

Scheme 3. Dehydrogenative coupling of benzylic and aldehydic $\mathrm{C}-\mathrm{H}$ bonds. collaborators performed an interesting $\mathrm{C}-\mathrm{H}$ benzylic functionalization using $\mathrm{HCl}$ as a precursor of $\mathrm{Cl}$ radical in a stop-flow microtubing (SFMT) reactor irradiated by blue lights (Scheme 4). ${ }^{[21]}$ The success of this photoredox functionalization laid on the microtubing reactor, in which $\mathrm{HCl}$ cannot evaporate, and consequently, escape from the reaction.

In 2019, Rueping and collaborators ${ }^{[22]}$ reported an elegant use of dual Nickel/photoredox catalysis for benzylic arylation, employing a diaryl ketone as both photosensitizer and a HAT agent. This approach fully benefits from the peculiar properties of diaryl ketones related to their accessible triplet excited state. 4,4'Dichlorobenzophenone was applied as a photocatalyst, to serve both as hydrogen-atom-transfer and as electron-transfer agent (Scheme 5). The reaction, with respect to the structure of the bromo-containing substrates, gave good to excellent yields. However, iodo-analogues exhibited much lower reactivity (39\% NMR yield). This was surprising because the aryl-I bond should be more reactive towards oxidative additions compared to the aryl-Br bond. To justify this result, Rueping's group, in line with the studies reported by Molander's group,${ }^{[17]}$ proposed an additional propagation pathway where the oxidative addition between haloarene and $\mathrm{Ni}^{0}$ species happens first. In the case of bromoarenes, this is followed by an energy-transfer step from the triplet state of the photocatalyst to $\mathrm{Ni}^{\mathrm{II}}$ species II (Scheme 5D) leading to an excited state, which undergoes subsequent homolysis to give $\mathrm{Ni}^{\mathrm{I}}$ III and a halide radical.

The halide radical can then abstract a hydrogen atom from the H-donor. This assumption was confirmed by addition of tetrabutyl ammonium bromide (TBAB) in the reaction mixture allowing for the ligand exchange to form $\mathrm{Ar}-\mathrm{LnNi}{ }^{\mathrm{II}}-\mathrm{Br}$ from $\mathrm{Ar}-\mathrm{Ni}^{\mathrm{iI}}-\mathrm{I}$ (Scheme 5D) which resulted in an increase of the yield.

As described above, the activation of benzylic compounds via HAT generates a carbon centred radical. This has trigonal planar geometry $\left(s p^{2}\right)$, thus the following functionalization will give a racemic mixture of the product. Nonetheless, chiral ligands have been successfully applied towards the enantioselective benzylic $\mathrm{C}-\mathrm{H}$ functionalization. Gong's group $^{[23]}$ reported an elegant enantioselective synthesis

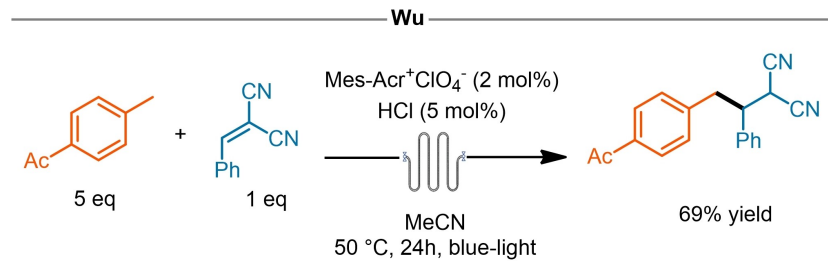

Scheme 4. $\mathrm{HCl}$ as a HAT catalyst precursor in a microtubing reactor. 

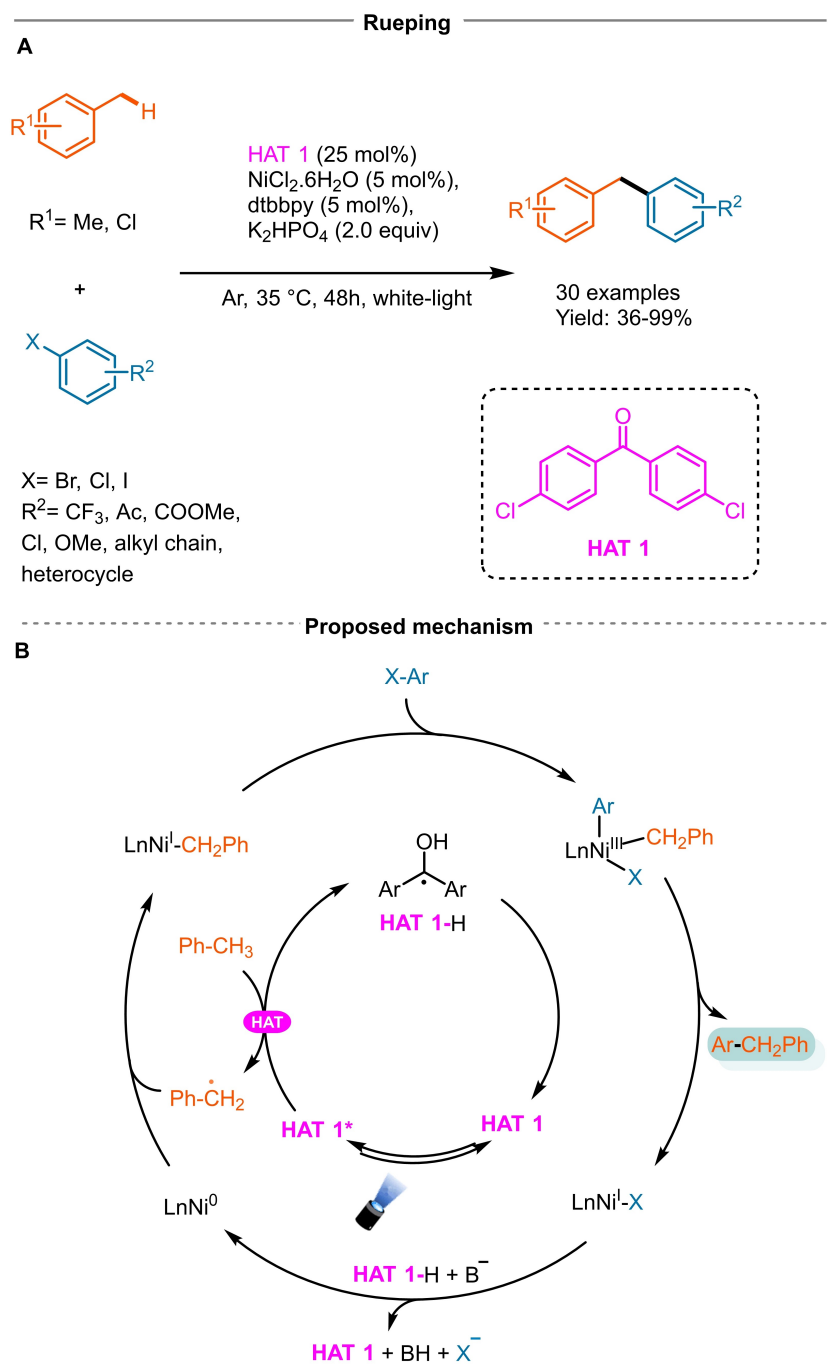

Selected scope

C

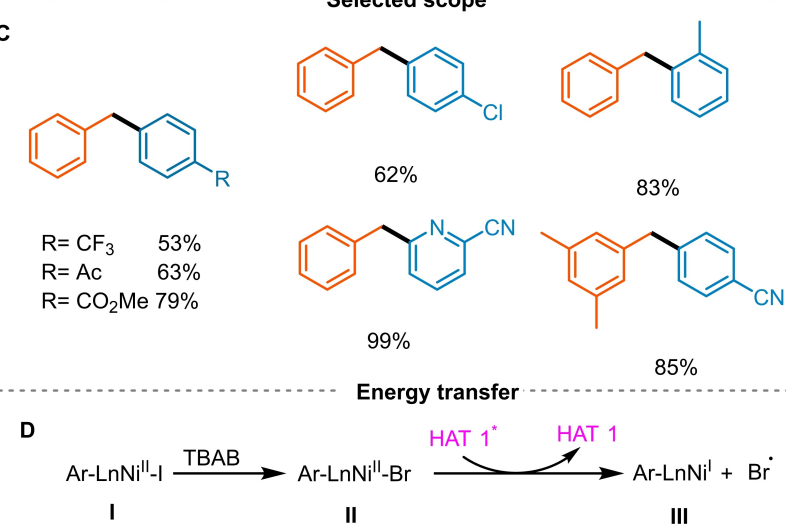

Scheme 5. The dual role of benzophenone in visible lightNickel photoredox catalysed $\mathrm{C}-\mathrm{H}$ arylation.

of chiral benzylic molecules by making use of the chiral bisoxazoline L2 (Scheme 6). Mechanistically, the photocatalyst is responsible for the generation of the reactive species whilst, by coordination with imine substrate, the metal (and chiral ligand) stabilizes the

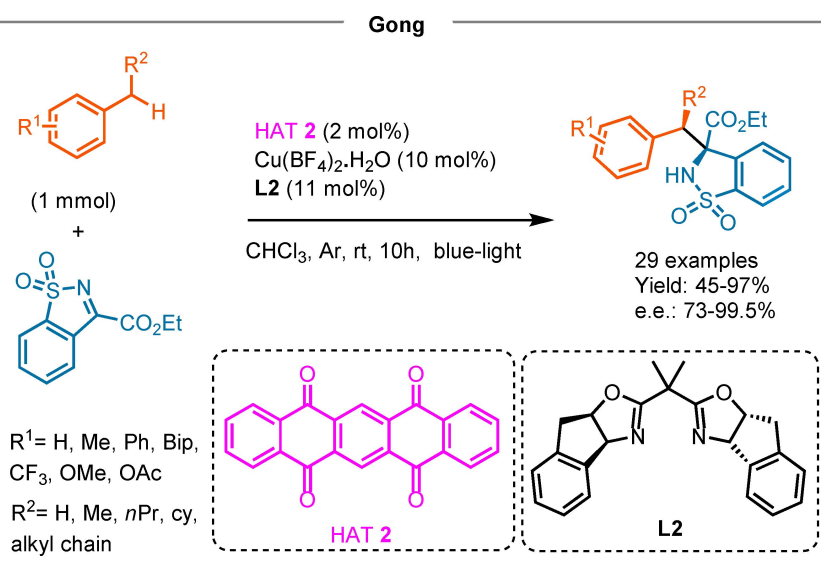

Scheme 6. Stereoselective $\mathrm{C}\left(s p^{3}\right)-\mathrm{H}$ functionalization of benzylic hydrocarbons.

generated radical and directs the regio- and stereoselectivity of the cross coupling. This synergy between the chiral $\mathrm{Cu}$ salt and HAT 2, led to the functionalization of the inactive benzylic $\mathrm{C}-\mathrm{H}$ bond with good to excellent regioselectivity (up to $>50: 1$ ) and enantioselectivity (up to $99.5 \%$ ), providing an economic and rapid construction of optically active compounds from basic chemical feedstocks.

In the same year, the group of Murakami showed a different application of benzophenone derivatives in the photo-induced carboxylation of toluene derivatives. $^{[2]}$ They presented a direct access to carboxylic acids from readily available hydrocarbon molecules, when irradiated with UV light in the presence of an aromatic ketone, a nickel complex, and potassium tert-butoxide (Scheme 7). The triplet photoexcited state of HAT 3 is more electrophilic than nucleophilic. This leads to the abstraction of a hydrogen from the most electron rich $\mathrm{C}-\mathrm{H}$ bonds. The reaction worked well with an electro-donating group on the benzene ring, but the yield decreased in the presence of halogens. On the other hand, no carboxylic acids were produced in case of 4-dimethylamino-, 4methylthio-, 4-cyano-, and 3-benzoyltoluenes.

In 2018, the group of Kanai designed a new HAT catalyst within the sulfonamide class, which, in combination with an iridium photocatalyst, could perform a selective arylation of the $\mathrm{C}\left(s p^{3}\right)$ benzylic position. ${ }^{[25]}$ Sulfonamides have proved to be a successful class of HAT reagents due to three main reasons: 1) the BDEs $(\mathrm{N}-\mathrm{H})$ of sulfonamides are within the proper range $\left(90-100 \mathrm{kcal} / \mathrm{mol}\right.$ vs $\mathrm{C}\left(s p^{3}\right)-\mathrm{H}=91 / 105 \mathrm{kcal} /$ mol); 2) the BDE value can be adjusted by changing the sulfur or nitrogen substituents; 3) sulfonamides are very easy to synthesize. As shown in scheme 8 , the catalytic cycle starts with excitation of the photocatalyst upon irradiation followed by single electron reduction by the deprotonated sulfonamide. The newly formed $\mathrm{N}$-centred radical activates the substrate ben- 


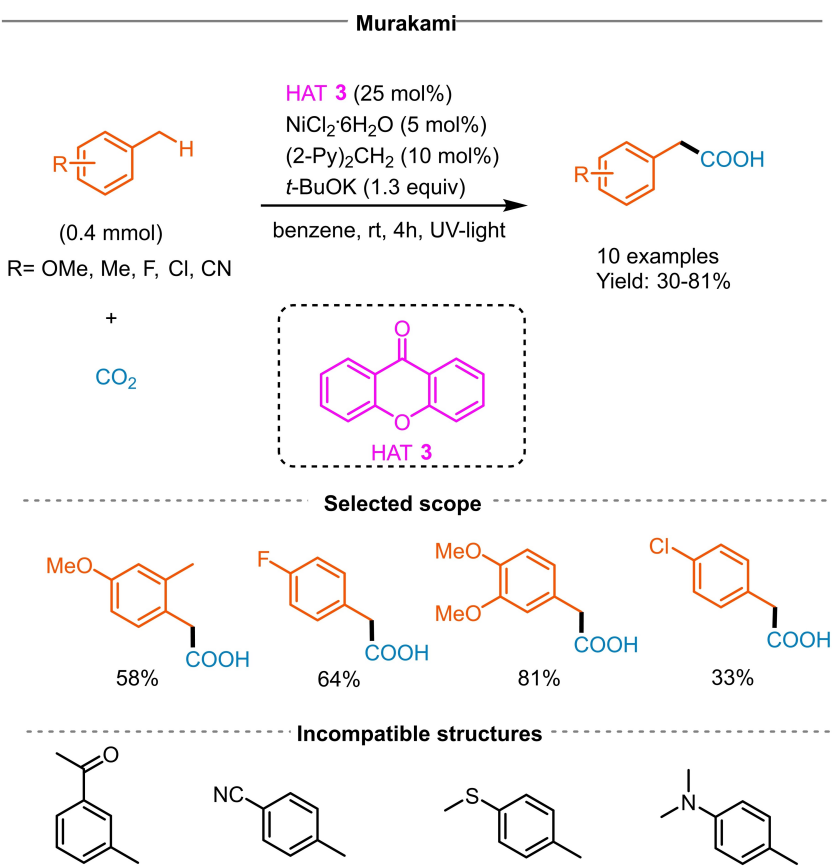

Scheme 7. Carboxylation of benzylic $\mathrm{C}-\mathrm{H}$ bonds with $\mathrm{CO}_{2}$ induced by light/ketone/Nickel.

zylic position via HAT. Meanwhile, the photocatalyst is regenerated by SET with 1,4-dicyanobenzene to form a phenyl radical anion. This can react with the benzyl radical and provide the final product after elimination of cyanide anion. The latter deprotonates the sulfonamide closing the HAT cycle.

The reaction conditions were successfully applied to the arylation of both cyclic and acyclic benzylic ethers. Moreover, the scope could be extended to benzyl alcohols and corresponding TBS analogues.

\section{2. $\mathrm{C}\left(s p^{2}\right)-\mathrm{O}$ and $\mathrm{C}\left(s p^{3}\right)-\mathrm{O}$ Bond Formation}

Oxygen-bearing groups (hydroxyl, ether and carbonyl) are important motifs in many pharmaceutical compounds and bioactive molecules ${ }^{[26]}$ as well as intermediates in cosmetic, agrochemical and polymer industry. ${ }^{[27-28]}$ Many oxidative processes have been developed in the past, but most of these procedures suffer from some limitations such as use of stoichiometric amounts of toxic $\mathrm{Cr}(\mathrm{VI}),{ }^{[29]} \mathrm{Mn}(\mathrm{IV})^{[30]}$ and $\mathrm{Se}(\mathrm{IV})^{[31]}$ reagents, or transition metal catalysts in combination with an excess of oxidants. ${ }^{[32]}$ Moreover, most of them do not tolerate heterocycles due to the strong coordination of heteroatoms to the metal catalysts and are performed at high temperature. In this section we will highlight the contribution of photochemical transformations for the oxidation of benzylic carbon resulting in mild conditions and a broad scope.

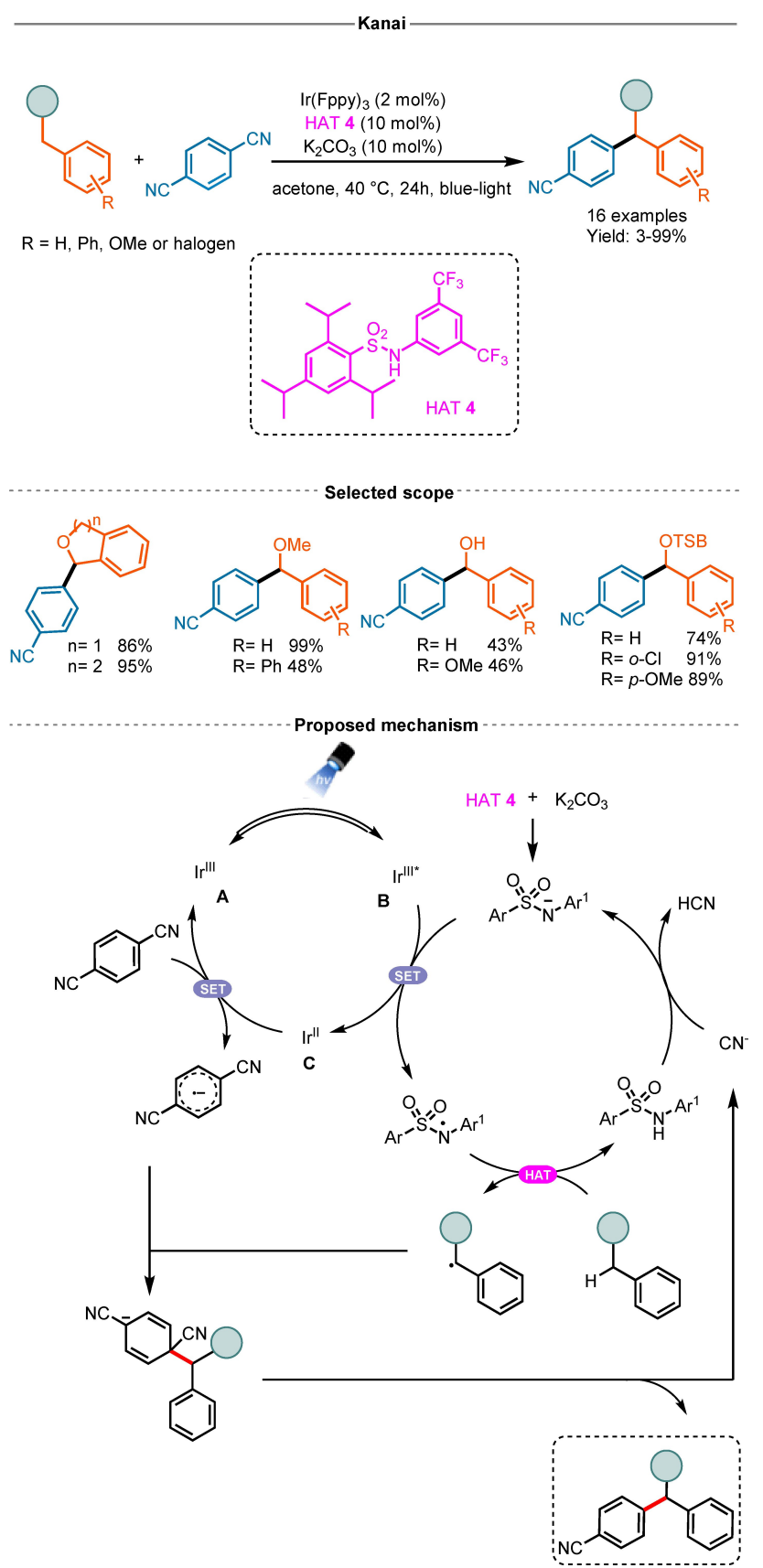

Scheme 8. Sulfonamides as new hydrogen atom transfer (HAT) catalysts for photoredox benzylic $\mathrm{C}-\mathrm{H}$ arylation.

\subsection{1. $\mathrm{O}_{2}$ as Oxygen Source}

In the last decade, several publications demonstrated molecular oxygen to be a valid alternative in oxidative processes. Moreover, inherent characteristics contributed to its success being a safe, readily available, costefficient, and environmental friendly oxidant. ${ }^{[33]}$

Lei and collaborators developed a mild route to obtain aryl ketones implementing photoredox catalysis using $\mathrm{O}_{2}$ oxidation (Scheme 9). ${ }^{[34]}$ The photocatalyst is responsible for the activation of the benzylic substrate 


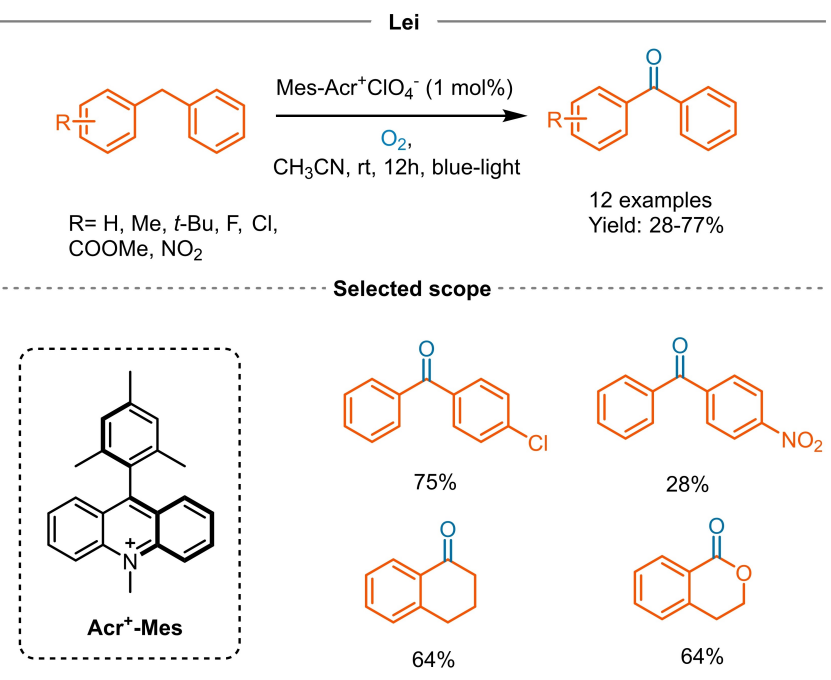

Scheme 9. Visible light mediated efficient oxidative benzylic $\mathrm{C}\left(s p^{3}\right)-\mathrm{H}$ to ketone derivatives obtained using $\mathrm{O}_{2}$.

through single-electron transfer to afford a radical cation which is intercepted by an oxygen species. In this photocatalytic reaction, oxygen acts both as oxidant and as oxygen source. The latter was proven via ${ }^{18} \mathrm{O}$ labelling experiments. The 9-mesityl-10-methylacridinium ion photocatalyst (Mes- $\mathrm{Acr}^{+} \mathrm{ClO}_{4}^{-}$) was found to give the best yields. The reaction showed good selectivity with methylated substrates affording oxidation on the diarylmethylene only. Nonetheless, both 1,2,3,4-tetrahydronaphthalene and isochromane gave the corresponding ketones in good yields. The reaction could tolerate electron-withdrawing substituents such as ester and ketone but a dramatic decrease in yield occurred with a nitro group. Chlorinated substrates afforded the desired products, allowing scope for further functionalization.

In 2017, Jiang's group extended the scope to alkylsubstituted benzyl substrates ${ }^{[35]}$ which has so far been limited to $p$-methoxy-substituted analogues. ${ }^{[36]}$ They employed an in-house developed metal-free photocatalyst ${ }^{[37]}$ based on the dicyanopyrazine (DPZ) core (Scheme 10). Direct oxidative activation of benzylic $\mathrm{C}\left(s p^{3}\right)-\mathrm{H}$ cannot be achieved by DPZ due to redox mismatch $\left[\mathrm{E}_{\mathrm{t}}\left(\mathrm{S}^{*} / \mathrm{S}^{-}\right)=0.91 \mathrm{~V}\right.$ vs $\mathrm{SCE}$ in $\mathrm{CH}_{2} \mathrm{Cl}_{2} ; \mathrm{E}_{\text {ox }}^{0}$ (ethylbenzene) $=2.14$ vs $\mathrm{SCE}$ in $\left.\mathrm{CH}_{3} \mathrm{CN}\right]$. As expected, their first attempt to oxidize 1,2,3,4tetrahydronaphthalene failed. To overcome this issue a dual catalyst involving $N$-hydroxyphthalimide (NHPI) was employed. Oxidation of NHPI $\left(\mathrm{E}_{\mathrm{red} 1 / 2}=+0.78 \mathrm{~V}\right.$ vs $\mathrm{SCE}$ in $\mathrm{CH}_{3} \mathrm{CN}$ ) by the exited state of DPZ generates phthalimide- $N$-oxyl (PINO) which is able to undergo HAT reaction with the benzylic $\mathrm{C}\left(s p^{3}\right)-\mathrm{H}$ bond to form NHPI and the benzyl cation. Molecular oxygen once again covers the role of oxidant closing the photoredox cycle and oxygen source. Optimal conditions were found by screening different $N$ -

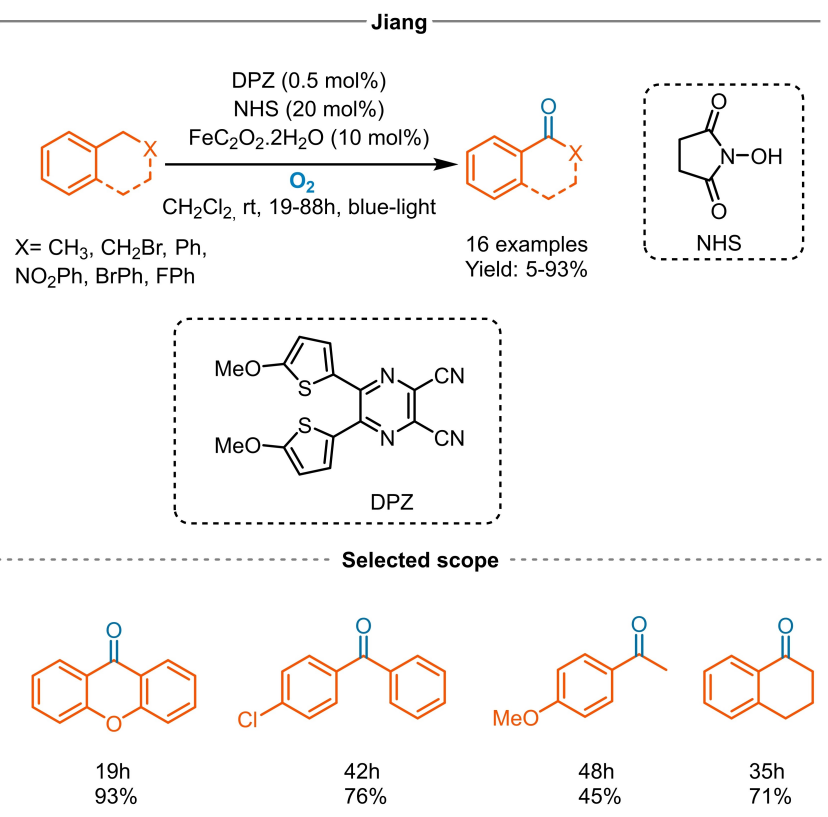

Scheme 10. Oxidation of benzylic $\mathrm{C}\left(s p^{3}\right)-\mathrm{H}$ bonds through $N$ hydroxymide and dicyanopyrazine.

hydroxyimides and additives. The best yield was obtained using $\mathrm{N}$-hydroxysuccinimide (NHS) and $\mathrm{FeC}_{2} \mathrm{O}_{2} \cdot 2 \mathrm{H}_{2} \mathrm{O}$. The benzyl alcohol is believed to be a possible intermediate, its conversion to the ketone was obtained successfully without the need of $\mathrm{FeC}_{2} \mathrm{O}_{2} \cdot 2 \mathrm{H}_{2} \mathrm{O}$. Benzylic methylenes with both electron-donating and -withdrawing substituents gave the corresponding ketones in good yields although a longer reaction time was required to reach full conversion. Noticeably, photooxygenation of (2-bromoethyl) benzene successfully afforded 2-bromo-acetophenone. Biphenyl-methane derivatives and xanthene were also amenable of oxidation. When toluene was used benzoic acid was obtained in low yield with traces of benzaldehyde.

Several other homogenous photocatalysts such as benzoquinones, ${ }^{[38]}$ anthraquinones, ${ }^{[39]}$ eosin $\mathrm{Y}^{[40]}$ and functionalized carbon $\operatorname{dots}^{[41]}$ have been successfully employed in the benzylic oxidation with molecular oxygen.

Cong's group reported the aerobic oxidation of benzyl ethers employing a dye-sensitized semiconductor as visible-light photocatalyst (Scheme 11) ${ }^{[42]}$ Metal oxide semiconductor heterogeneous photocatalysis is usually limited to UV light as the gap between the valence band and the conduction band requires high energy photons. This is essential to promote electrons and form electron/hole pair which are exploitable in redox reactions. This limitation can be overcome with dye-sensitized semiconductors where electrons are filled by the organic dye directly in the conduction band by harvesting visible-light. ${ }^{[43]}$ In the present case, 


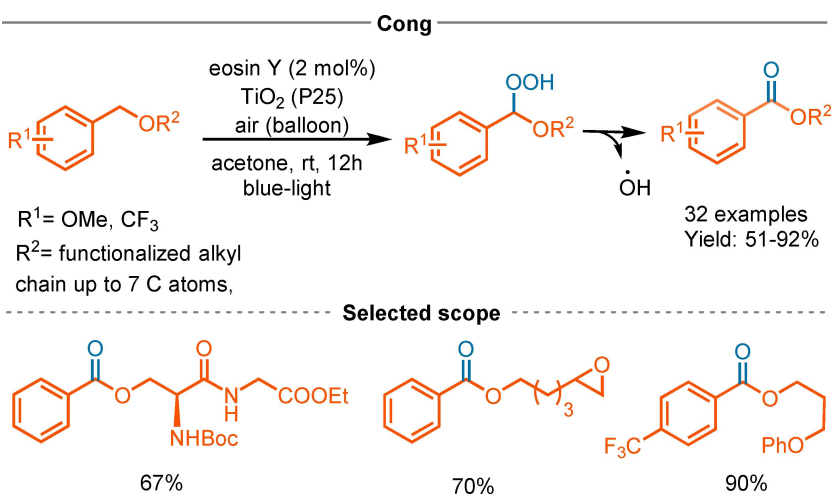

Scheme 11. Selective aerobic oxidation of benzyl ethers employing a dye-sensitized semiconductor.

screening of different combinations of semiconductor and dye resulted in P25-type $\mathrm{TiO}_{2}$ and eosin $\mathrm{Y}$ as the optimal match. The reaction conditions were effective regardless the steric hindrance of the alkyl groups, and several functional groups were well tolerated. Moreover, electron-donating and -withdrawing groups on the benzyl ring did not affect the yield. Oxygen protecting groups such as acetate, sulfonate, silyl ether, and acetal were not affected. Finally, the reaction conditions could be successfully applied to a variety of natural products, including dipeptides and monosaccharides, demonstrating high selectivity. In depth mechanistic investigation suggested the initial formation of a benzyl peroxide, with concomitant degradation of the organic dye, followed by photoinduced radical processes between substrate and solvent, leading to the formation of the final product.

In a recent publication from Ren and collaborators $^{[44]} \mathrm{AgI} / \mathrm{BiVO}_{4}$ composites have been employed in the photooxidation of alkylbenzenes and benzyl ethers (Scheme 12). $\mathrm{AgI}$ and $\mathrm{BiVO}_{4}$ are both exited by visible-light and are able, to a certain extent, to catalyse the reaction giving the oxidation product in low yields or traces, respectively. A mixture of the two did not result in a noticeable improvement. Contrarily, $\mathrm{AgI} / \mathrm{BiVO}_{4}$ composites prepared via in situ depositionco-precipitation, afforded the desired product in good yield. Simultaneous excitation of $\mathrm{AgI}$ and $\mathrm{BiVO}_{4}$ by visible-light causes promotion of electrons from the valance band to the conductive band in both materials. Photoelectrons from $\mathrm{BiVO}_{4}$ conductive band flow in the valence band of $\mathrm{AgI}$ with fast recombination with the hole. This interaction facilitates the single-electron oxidation of the organic substrate to radical cation, by the $\mathrm{VB}$ of $\mathrm{BiVO}_{4}$ and the reduction of $\mathrm{O}_{2}$ to superoxide radical anion via SET from the VB of AgI. The newly formed charged radical species can first react to form hydroperoxidate intermediate and finally the carbonyl product. The heterogeneous nature of the catalytic process was confirmed by leaching test and mercury

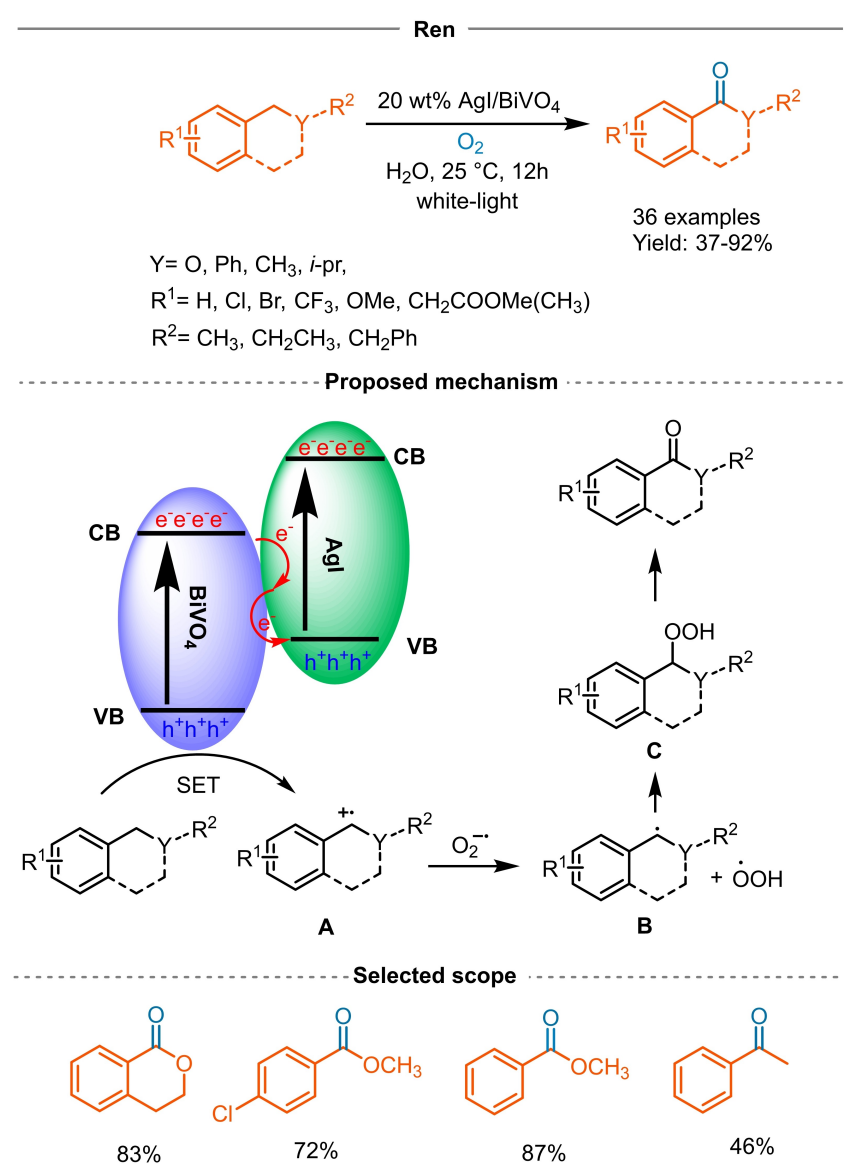

Scheme 12. Selective aerobic oxidation of $\mathrm{C}\left(s p^{3}\right)-\mathrm{H}$ bonds catalysed by heterogeneous $\mathrm{AgI} / \mathrm{BiVO}_{4}$ catalyst.

poisoning experiments. Moreover, the catalyst could be easily separated from the reaction mixture and recycled without loss in activity. Notably, water was found to be the optimal solvent. Benzyl esters and ketones were obtained in good to excellent yields regardless the electronic effect of the substituents.

Finally, a recent report regarding photo/enzymatic $\mathrm{C}-\mathrm{H}$ hydroxylation ${ }^{[45]}$ is worth mentioning. Chemoenzymatic catalysis ${ }^{[46]}$ has promised to be a turning point in enantioselective transformations. Nonetheless, in order to successfully combine organo- and biocatalysis in a one-pot process, additional optimization is required as enzymes usually operate optimally in a narrow range of conditions. Also, temperature, solvents, and additives required for the organocatalysed step should not cause enzyme inhibition. Scheidt's group ${ }^{[45]}$ found that visible-light photocatalysed benzylic oxidation is a good starting point as the reaction is usually run close to room temperature and water can be a compatible cosolvent (Scheme 13).

Photocatalysed oxidation and enzymatic reduction was first optimized independently. The choice of enzymatic system fell on KRED-P1-A04, a nicotinamide adenine dinucleotide phosphate $(\mathrm{NAD}(\mathrm{P}) \mathrm{H})$ 


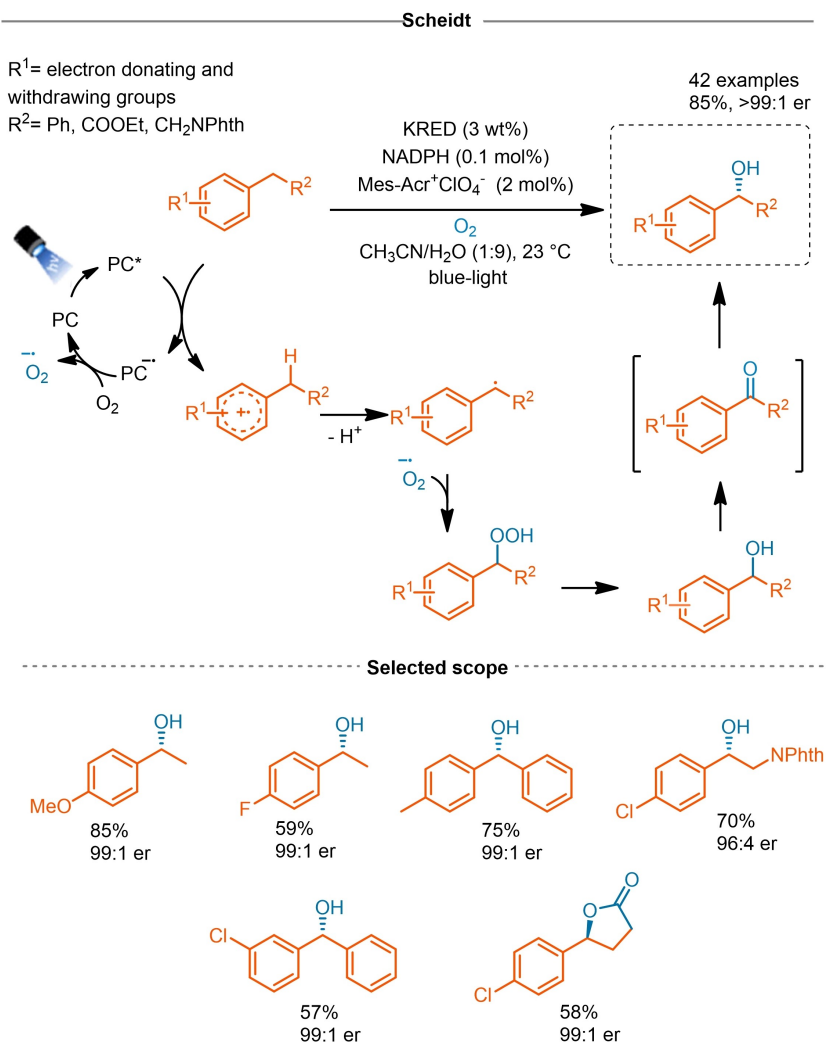

Scheme 13. Combined photoredox/enzymatic $\mathrm{C}-\mathrm{H}$ benzylic hydrocylations.

dependent ketoreductase (KRED) class that has already been proven to be compatible with photoredox conditions. Unfortunately, the initial attempt of a one-pot reaction revealed isopropyl alcohol (IPA), required for the regeneration of $(\mathrm{NAD}(\mathrm{P}) \mathrm{H})$, to be incompatible with photoredox conditions. The issue was promptly solved by opting for a two steps-process starting with the addition of IPA after the completion of the photooxidation and in the absence of irradiation. The optimized reaction conditions tolerated a variety of substrates enabling the synthesis of aryl-alkyl alcohols, diarylmethanols, $\alpha$-hydroxy esters, and 1,2-amino alcohols in good yields and excellent enantiomeric ratio. Moreover, when phenyl tethered alkyl esters were reacted, the corresponding lactones were obtained.

\subsubsection{Alcohols as Oxygen Source}

Earlier we have described the synthesis of ketones through photooxygenation of benzylic $\mathrm{C}\left(s p^{3}\right)-\mathrm{H}$ developed by Lei and collaborators (Scheme 9). ${ }^{[34]}$ In 2016, the same group discovered that treating toluene derivatives under the same reaction conditions with methanol as solvent, could afford the corresponding methyl esters as product (Scheme 14). ${ }^{[47]}$ Addition of a sub-stoichiometric amount of $\mathrm{HCl}$ greatly increased

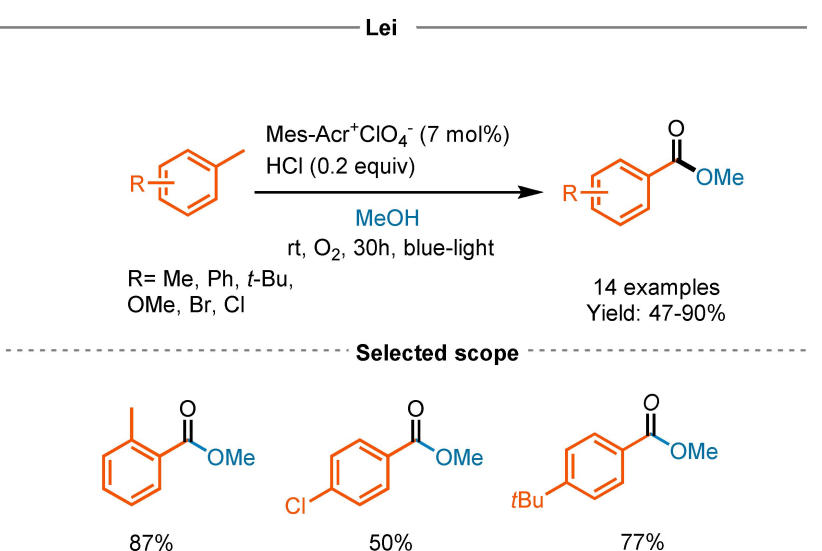

Scheme 14. Visible-light induced oxidative $\mathrm{C}\left(s p^{3}\right)-\mathrm{H}$ activation of methyl aromatics to methyl esters.

the yield and a variety of analogues could be obtained in good yields, though strong electron-withdrawing substituents completely inhibited the reaction. Replacement of methanol with ethanol and isopropanol provided the products in low yield. The formation of the ester happens presumably as last step via oxidative coupling with the benzaldehyde generated by photocatalytic oxidation of toluene.

Recently, Yoon's lab has developed a method for the photocatalysed direct $\mathrm{C}-\mathrm{H}$ alkoxylation for the synthesis of benzyl ethers. ${ }^{[48]}$ They managed to avoid the over oxidation of the benzyl position by working in inert atmosphere and by addition of a terminal oxidant. Best results were obtained using $1 \mathrm{~mol} \%$ of $[\operatorname{Ir}(\mathrm{dF}-$ $\left(\mathrm{CF}_{3}\right)$ ppy $)_{2}\left(5,5^{\prime}-\mathrm{dCF}_{3}\right.$ bpy $\left.)\right] \mathrm{PF}_{6}, 1.2$ equiv. of $\mathrm{Cu}(\mathrm{TFA})_{2}$ $(\mathrm{MeCN}), 2$ equiv. of alcohol in acetonitrile and irradiation at $427 \mathrm{~nm}$ (Scheme 15). The reaction tolerates a wide range of differently decorated alcohols and side chains, including alkene, alkyne, aldehyde, nitrile, boronate esters, and both basic- and acid-sensitive groups. However, the scope was limited to para alkoxy-phenyl substrates. This can be explained by analysing the catalytic cycle. Copper salts, apart from regenerating the photocatalyst, play a role in the oxidation of the benzyl radical to form a cation stabilized through the formation of a quinone methide intermediate. Nonetheless, this feature guaranteed high

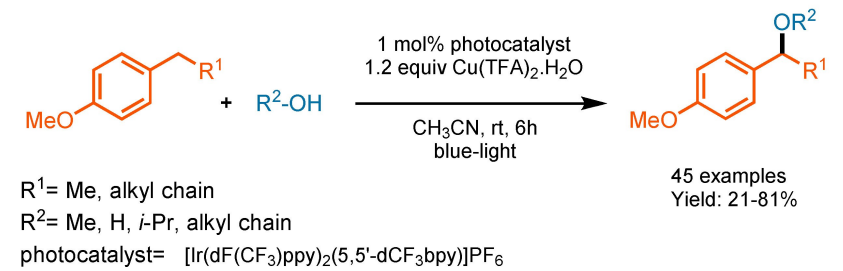

Scheme 15. Site-selective alkoxylation of benzylic C-H bonds. 
selectivity also in the presence of other weak $\mathrm{C}-\mathrm{H}$ bonds otherwise susceptible of oxidation. Finally, the benzyl position of electron-rich heterocycles was also amenable to functionalization.

\subsubsection{Intramolecular Oxidation}

In a pioneering work in the field of benzylic $\mathrm{C}\left(s p^{3}\right)-\mathrm{H}$ functionalization, Pandey and collaborators successfully developed a novel intramolecular cyclo-etherification reaction ${ }^{[36]}$ exploiting a cyanoaromatic-catalysed photoredox process (Scheme 16). This represented one of the first examples of benzylic $\mathrm{C}-\mathrm{H}$ oxidation under photocatalytic conditions. Recently, the same group upgraded the catalytic system enabling the synthesis of both $O$ - and $N$-heterocycles. ${ }^{[49]}$ They employed a ruthenium photocatalyst and Selectfluor

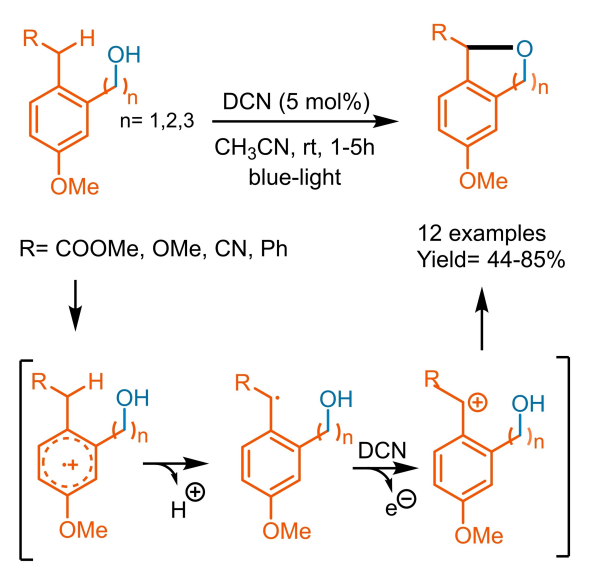

Scheme 16. Regioselective benzylic $\mathrm{C}-\mathrm{H}$ activation for $\mathrm{C}-\mathrm{O}$ bond formation.

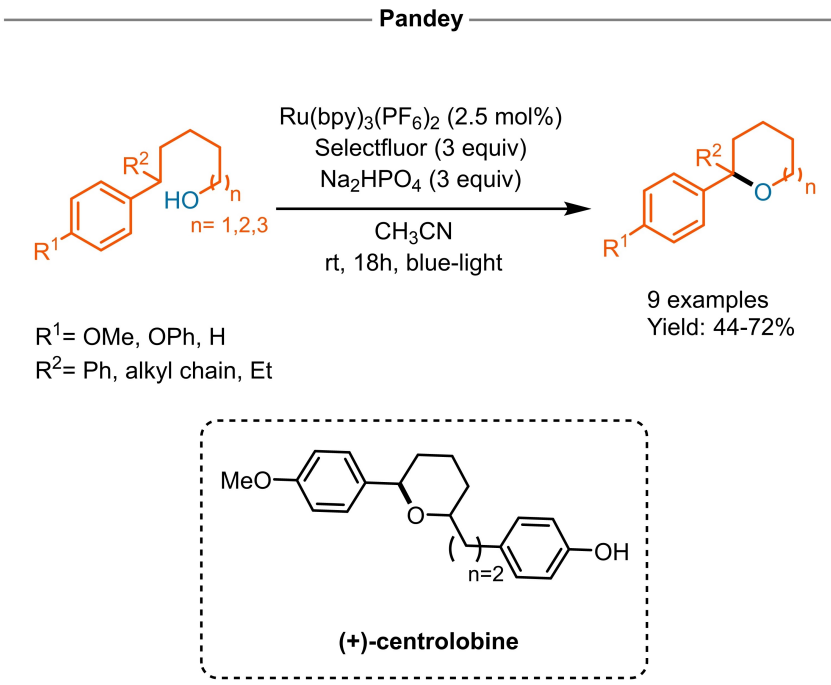

Scheme 17. Benzylic C-H activation for $\mathrm{C}-\mathrm{O}$ bond formation. for the subsequent oxidation/HAT of the benzylic substrate to form the benzyl carbocation which was trapped intramolecularly by the $O$ - or $N$-nucleophile (Scheme 17). The reaction afforded 5-, 6- and 7membered rings in good yields. Tertiary benzylic positions were also amenable to functionalization. Chiral centres adjacent to the alcohol moiety were not affected by the reaction conditions as demonstrated by the diastereoselective synthesis of the antibacterial $(+)$-centrolobine.

\section{3. $\mathrm{C}\left(s p^{3}\right)-\mathrm{N}$ Bond Formation}

During the last two decades, several approaches have been applied for the direct $\mathrm{C}-\mathrm{H}$ amination chemistry. $^{[50]}$ However, the majority of these uses either costly and toxic transition metal catalysts ${ }^{[51]}$ or requires strong oxidizing agents. ${ }^{[52]}$ In 2015, Pandey and Laha applied photoredox catalysis to comply with the oxidative activation of the benzylic $\mathrm{C}\left(s p^{3}\right)-\mathrm{H}$ bond towards $\mathrm{C}-\mathrm{N}$ bond formation employing amides as nitrogen source. ${ }^{[53]}$ As shown in Scheme 18, the photocatalytic cycle starts with the excitation of 9,10dicyanoanthracene (DCA) photocatalyst to its singlet state. This is involved in the generation of radical $\mathbf{C}$ that is able to activate the benzyl position via HAT.

Single electron oxidation of the newly formed benzylic radical by the photocatalyst exited state gives a carbocation readily available to be trapped by the amide. Molecular oxygen acts as sacrificial oxidant for
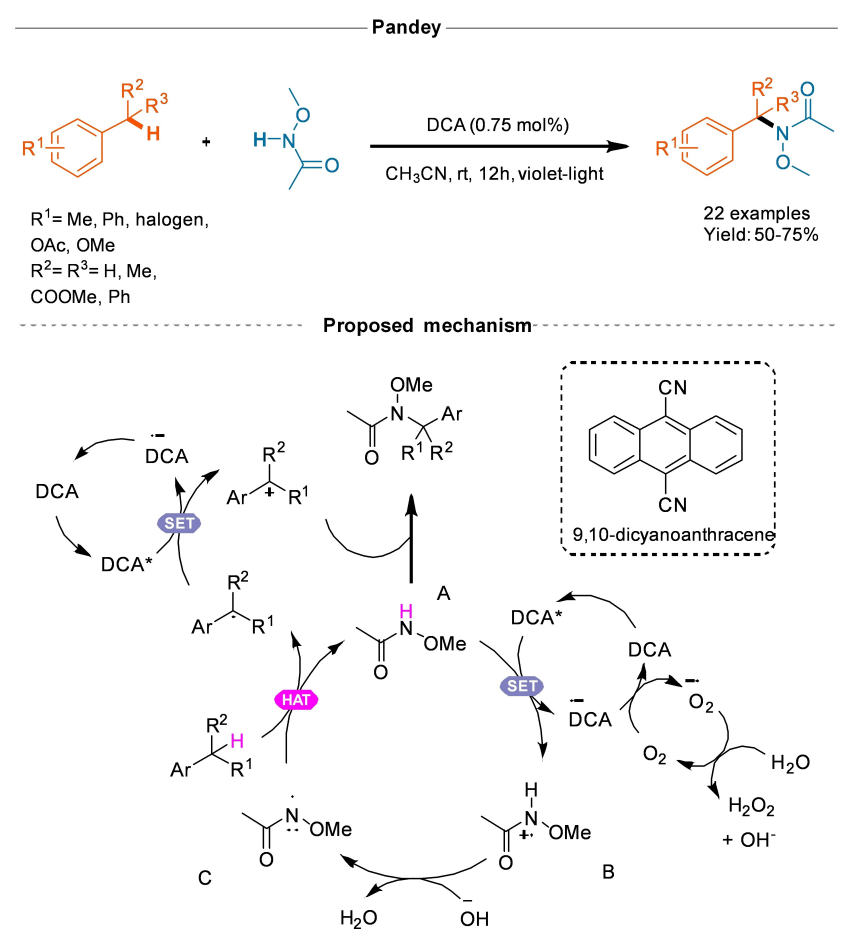

Scheme 18. Direct benzylic amination reaction by CDC. 
the regeneration of the photocatalyst. The incorporation of the HAT reagent in the final product further increases the atom economy of the reaction. The procedure has a broad substrate scope retaining the regioselectivity of the benzylic position, thus demonstrating the applicability of the protocol for the transformation of inexpensive alkylarenes.

A similar approach could be applied for the synthesis of nitrogen heterocycles. $O$-Benzyl- $N$-methoxybenzamides bearing both the $N$-nucleophile and the benzylic position should undergo intramolecular dehydrogenative cross-coupling to form isoindolinone cyclization products. In 2018, Xiao's group ${ }^{[54]}$ proved this assumption to be true treating the starting material with Selectfluor as HAT reagent and Eosin Y as photocatalyst under irradiation with blue LED (Scheme 19).

They further exploited the HAT/photoredox synergetic catalytic system by simple addition of oxygen to the reaction mixture. Molecular oxygen acted as terminal oxidant, regenerating the photocatalyst being reduced to radical anion. As shown in the reaction mechanism, isoindolinone is still susceptible of hydrogen abstraction by Selectfluor with the formation of a benzyl radical that when intercepted by the $\mathrm{O}_{2}$ radical anion could form a hydroperoxide intermediate. Reduction of the latter by another molecule of isodolinone gives 3-hydroxyisoindolinones. The desired oxygenated products were obtained in moderate to good yields and a diverse substitution pattern on both the amide and benzyl moieties were well

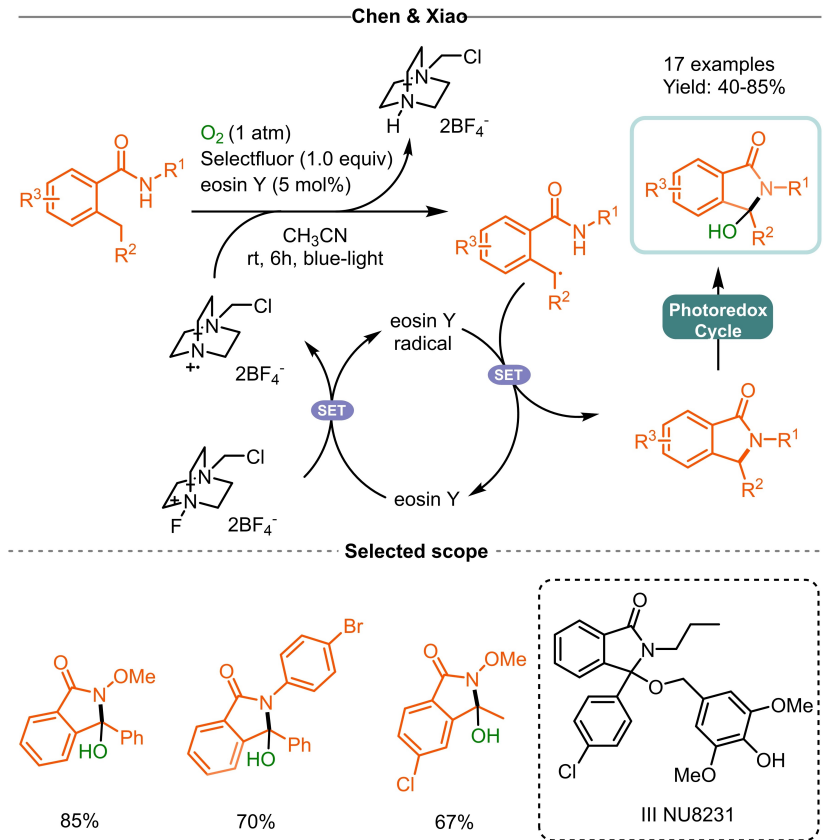

Scheme 19. Eosin $\mathrm{Y}$ as a redox catalyst and photosensitizer for sequential benzylic $\mathrm{C}-\mathrm{H}$ amination and oxidation. tolerated. Noticeably, comparable yields were obtained under sunlight irradiation. Finally, the protocol was successfully applied to the synthesis of III NU8231 inhibitor of the MDM2-p53 interaction.

In the previous section, we have described Pandey's work on Selectfluor mediated HAT/photoredox benzylic $\mathrm{C}\left(s p^{3}\right)-\mathrm{H}$ oxidation (Scheme 17) ${ }^{[49]}$ They have also applied the same strategy for the synthesis of cyclic amines and sulfamidates via $\mathrm{C}\left(s p^{3}\right)-\mathrm{H}$ amination (Scheme 20). A variety of electron-withdrawing substituents on the amine were compatible. Nonetheless, substitution on the benzylic ring was mainly restricted to $p$-methoxy. Phenyl and alkyl substitution on the benzylic position caused small reductions in yield. The catalytic system has also been successfully applied to the synthesis of the natural product (-)codonopsinine.

In the field of radical $\mathrm{C}-\mathrm{H}$ amination, azide synthesis has been thoroughly investigated in past decades ${ }^{[55]}$ given the great importance of azides as synthons and bioactive compounds. Greaney and collaborators implemented photoredox catalysis in the $\mathrm{C}-\mathrm{H}$ azidation using Zhdankin reagent (Scheme 21). ${ }^{[56]}$ Constructing on previous results with alkene azidation ${ }^{[57]}$ they started the optimization using $\mathrm{Cu}$ (dap) ${ }_{2} \mathrm{Cl}$ photocatalyst obtaining good yield. Noticeably, more common iridium and ruthenium photocatalysts failed in providing the desired product. Mechanistic investigation confirmed the radical nature of the reaction as it was completely inhibited upon addition of radical scavengers such as 2,2,6,6tetramethyl-1-piperidinyloxyl (TEMPO) and 2,6-ditert-butyl-4-methylphenol (BHT).

The azide radical is believed to be formed upon reduction of Zhdankin reagent by the exited state of $\mathrm{Cu}(\mathrm{dap})_{2} \mathrm{Cl}$. This radical is then able to activate the benzylic $\mathrm{C}-\mathrm{H}$ bond by abstraction of a hydrogen with the formation of hydrazoic acid. The benzyl radical can therefore propagate the reaction abstracting an azide from the Zhdankin reagent with the formation of an iodane radical that can undergo HAT with the

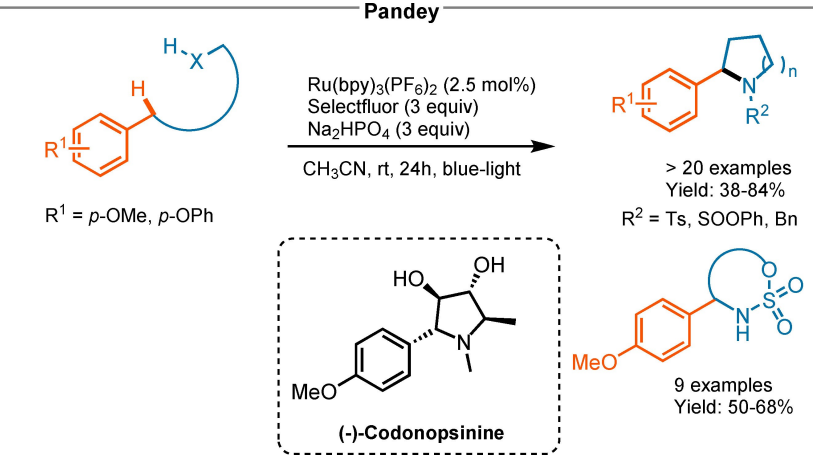

Scheme 20. Heterocyclization involving benzylic $\mathrm{C}\left(s p^{3}\right)-\mathrm{H}$ functionalization. 


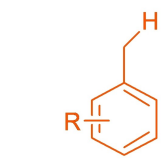

$\mathrm{R}=\mathrm{NO}_{2}, \mathrm{OMe}, \mathrm{CN}, \mathrm{Me}$, $\mathrm{Br}, \mathrm{I}, \mathrm{Cl}, \mathrm{F}, \mathrm{COOMe}$

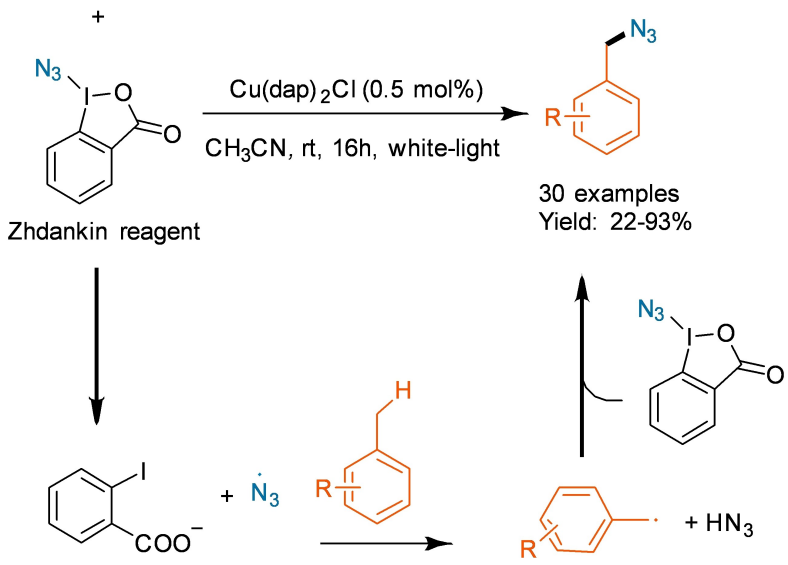

Scheme 21. Benzylic $\mathrm{C}-\mathrm{H}$ azidation using Zhdankin reagent and a copper photoredox catalyst.

benzylic substrate and form more benzyl radicals. The reaction conditions were applicable to a wide variety of benzylic substrates including indole and benzothiophene. Moreover, both electron-donor and -deficient substrates reacted efficiently. This direct azidation methodology was applied to the synthesis of the antiepileptic drug rufinamide without recourse to halogenation reagents or sodium azide in the synthetic sequence.

\subsection{C $\left(s p^{3}\right)-S$ Bond Formation}

Introduction of the trifluoromethylthio group $\left(\mathrm{SCF}_{3}\right)$ is extremely appealing as it can protect from cytochrome P450 enzymatic oxidation at the benzylic position. Moreover, increasing the lipophilicity can ease permeation through cell membrane with overall benefit to the pharmacokinetic profile. ${ }^{[58]}$ However, practical challenges hindered the development of this field as the only known methodology required a large excess of benzylic substrate, limiting its applicability to simple and cheap compounds. ${ }^{[59]}$ Direct HAT activation might fail to yield high selectivity in a substrate with multiple $\mathrm{C}-\mathrm{H}$ bonds that are characterized by comparable BDEs. This is generally the case while attempting latestage functionalization, especially of complex molecules. In 2019, Zhu and collaborators reported a protocol for the selective trifluoromethylthiolation of benzylic $\mathrm{C}\left(s p^{3}\right)-\mathrm{H}$ bonds under photocatalytic con- ditions (Scheme 22) ${ }^{[60]}$ Phth-SCF 3 (2-((trifluoromethyl)thio)isoindoline-1,3-dione) was chosen as the $\mathrm{SCF}_{3}$ source and the best results were obtained with 4CzIPN photocatalyst and $\mathrm{K}_{2} \mathrm{CO}_{3}$ in anhydrous acetonitrile with blue LEDs irradiation. Noticeably, HAT reagents were not required and the proposed mechanism spots some light on the outstanding selectivity. Upon irradiation of the photocatalyst, its exited state readily oxidizes the benzylic substrate to a radical cation. This undergoes irreversible intramolecular inner-sphere 1,2$\mathrm{HAT} /$ deprotonation resulting in a carbon centred radical which is subsequently trapped by the Phth$\mathrm{SCF}_{3}$ or its radical anion formed in the reductive side of the photocatalytic cycle.

\section{5. $\mathrm{C}\left(s p^{3}\right)$-Halogen Bond Formation}

Early studies on $\mathrm{C}\left(s p^{3}\right)-\mathrm{H}$ photocatalysed halogenations were conducted by Tan and collaborators (Scheme 23). ${ }^{[61]}$ They reported the bromination of $\mathrm{C}\left(s p^{3}\right)-\mathrm{H}$ benzylic and aliphatic bonds employing $\mathrm{CBr}_{4}$ both as a halogen source and a hydrogen abstraction species through formation of a $\mathrm{CBr}_{3}$ radical by the oxidative quenching of Eosin Y. Morpholine and water were found to be necessary as variation

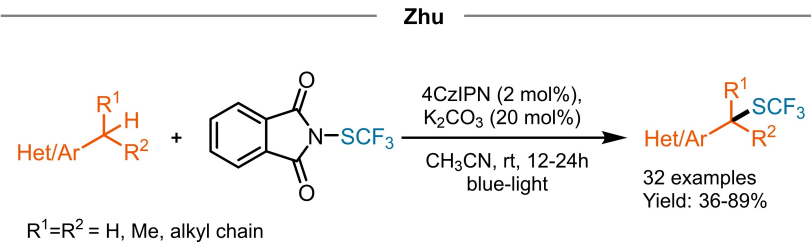

Scheme 22. Trifluomethylthiolation of benzylic C-H bonds.

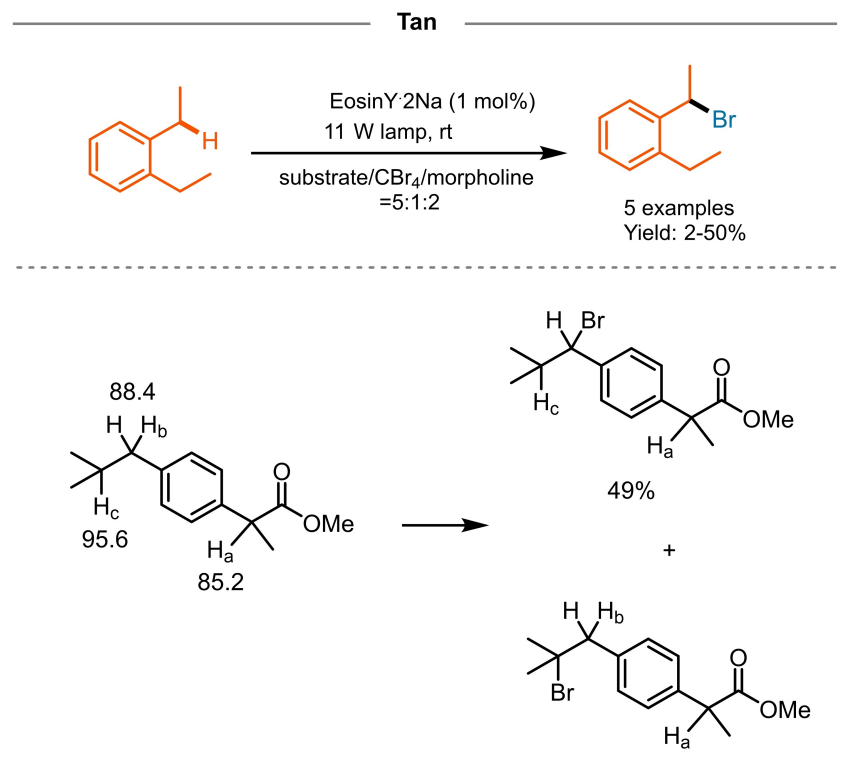

Scheme 23. Selective bromination of $\mathrm{C}\left(s p^{3}\right)-\mathrm{H}$ bonds. 
caused lower yields. The former acts as additional HAT reagent, via the generation of long-lived $\mathrm{N}$ radicals. The biphasic system exerts a thermodynamic pull by removing bromide anions in the form of protonated morpholine-bromide complexes, thus in turn favouring the dissociation of $\mathrm{CBr}_{4}$. The reaction showed unprecedented selectivity as exemplified in the bromination of estrone.

More recently, $\mathrm{Wu}$ and collaborators adopted a similar strategy for the selective fluorination of benzylic $\mathrm{C}-\mathrm{H}$ bonds (Scheme 24). ${ }^{[62]}$ Once again, the oxidative cycle of a photocatalyst was exploited for the activation of the halogen source, specifically Selectfluor. Upon reduction by the exited state of the photocatalyst (i.e. 9-mesityl-10-methylacridinium), Selectfluor splits into fluoride anion and a radical dication. The latter can undergo HAT with activation of the benzylic site of the substrate. The benzyl radical can then afford the fluorinated product via a direct reaction with another molecule of Selectfluor or by engaging the photocatalytic cycle to form a carbocation subsequently trapped by $\mathrm{F}^{-}$. Serving as both a halogen source and as an $N$-radical HAT reagent, Selectfluor demonstrated to be an efficient and versatile reagent, allowing the fluorination of a variety of benzylic substrates in moderate to good yields. The electrophilic nature of the $N$-radical dication led to better results in the presence of electron-donating substituents, as electron-rich $\mathrm{C}-\mathrm{H}$ bonds are more easily cleaved. Noticeably, when diphenylmethanes were treated in the same conditions, diaryl ketones were the only product.

\section{Electrochemical Benzylic C(sp $\left.p^{3}\right)-\mathrm{H}$ Functionalization}

In the previous section, we have analysed catalytic cycles involving single-electron redox reactions triggered by visible-light using a photosensitizer. Although this has been demonstrated to be a valid alternative for the use of stoichiometric oxidants, electrochemistry

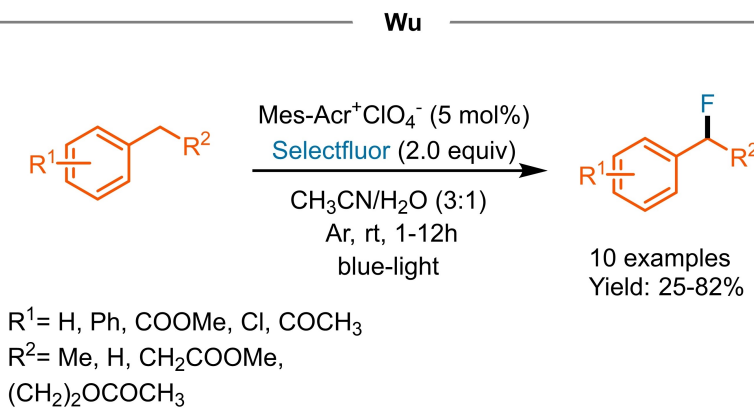

Scheme 24. Reducing ability of $\mathrm{Acr}^{+}$-Mes for fluorination of benzylic $\mathrm{C}\left(s p^{3}\right)-\mathrm{H}$ bonds under visible light irradiation. has emerged as another viable option for fine organic synthesis.

Direct exchange of electrons with the substrate, via the application of electrical potential, offers direct access to a redox equation easy to write on paper but still challenging in real life. Virtually, all coupling reactions involving an oxidative activation step could be run in electrochemical conditions, where the anode takes the burden of the "undesired surplus" of electrons. Intensive research has broadened the applicability of organic electrochemical processes providing access to untraveled pathways, ${ }^{[63]}$ benzylic $\mathrm{C}\left(s p^{3}\right)-\mathrm{H}$ oxidation-functionalization not being an exception. Nonetheless, new technologies and approaches come always with some challenges. In this section, we will address the scope and limitations of electrochemical benzylic $\mathrm{C}\left(s p^{3}\right)-\mathrm{H}$ functionalization by reviewing the latest findings on the topic, with special attention to the strategies chosen to overcome inherent issues of this approach.

\subsection{C $\left(s p^{3}\right)-C$ Bond Formation}

Anodic oxidation of the benzylic $\mathrm{C}\left(s p^{3}\right)-\mathrm{H}$ bond to form a reactive carbocation, able to be trapped by a nucleophile, is a widely accepted strategy for electrochemical functionalization. Nonetheless, this route is inevitably hampered whenever the nucleophile redox potential is lower than that of the starting material. Due to redox mismatch between the starting material and the coupling partner, oxidation of the latter will be favoured, and no coupling will occur. Pioneering work from Yoshida and collaborators ${ }^{[64]}$ addressed this major limitation of electrochemical functionalization by developing the so called "cation pool" method. Electrolysis of the starting material was carried out at low temperature in the absence of nucleophiles, allowing for the carbocations to accumulate at the anode. Addition of the nucleophile in non-oxidant conditions completed the one-pot two-step process. In 2016, this protocol was further optimized, by the same group ${ }^{[65]}$ through the addition of stabilizing reagents to avoid decomposition of highly unstable carbocations such as benzylic cations (Scheme 25). After a brief screening, diphenylsulfilimine was demonstrated to be a suitable stabilizing agent. The reaction worked efficiently with electron-rich benzylic substrates and a variety of nucleophiles.

When the difference in redox potential between the product (higher potential) and the starting material is sufficient, direct electrolysis can be carried out without the fear of any side reactions due to overoxidation. An example was provided in 2017 by Luo's group where they combined cross-dehydrogenative coupling with organocatalysis for the enantioselective alkylation of $N$-phenyl-tetrahydroisoquinoline ${ }^{[66]}$ (Scheme 26). The reaction was run in an undivided cell using a graphite 


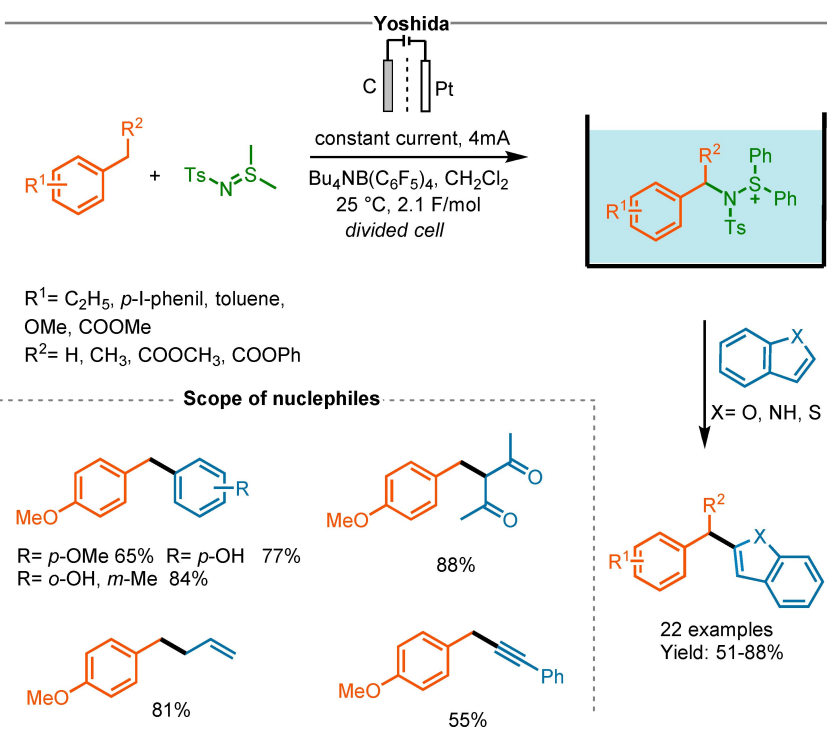

Scheme 25. Stabilized cation pool method for benzylic $\mathrm{C}-\mathrm{H}$ cross-coupling.

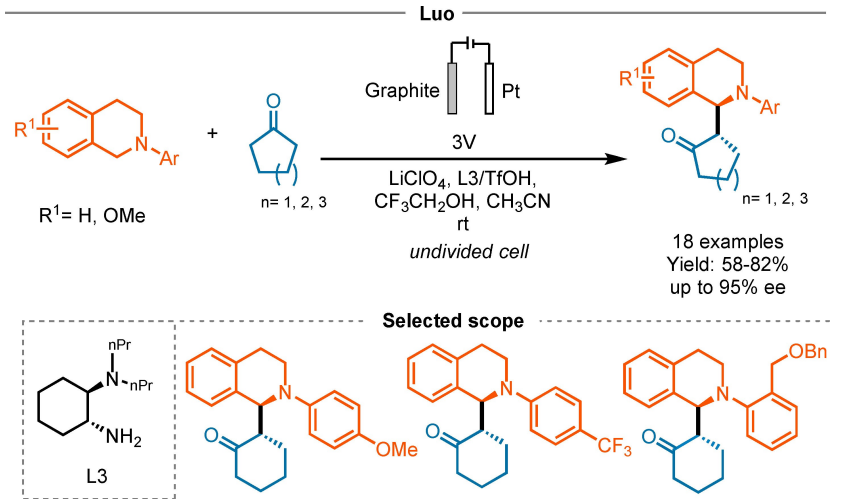

Scheme 26. Electrochemical asymmetric coupling of tertiary amines with ketones.

anode and a platinum cathode in acetonitrile and $\mathrm{LiClO}_{4}$ under nitrogen atmosphere and with a cell potential of 3.0 V. Enantiopure diamines were used as catalyst to activate

the ketone and to direct the capture of the reactive iminium intermediate formed at the anode by the oxidation of $N$-phenyl-tetrahydroisoquinoline.

Addition of a protic additive, the best being trifluoroethanol, was crucial to facilitate the capture of the iminium cation by the enamine, probably through formation of a more stable hemiaminal intermediate.

A recent example of one pot $\mathrm{C}\left(s p^{3}\right)-\mathrm{C}\left(s p^{3}\right)$ dehydrogenative coupling between xanthenes and ketones has been reported by $\mathrm{Li}$ and collaborators (Scheme 27). ${ }^{[67]}$ Xanthene derivatization has been widely investigated ${ }^{[68]}$ due to their interesting biological activities and the ease at which they undergo functionalization under oxidative conditions. The latter

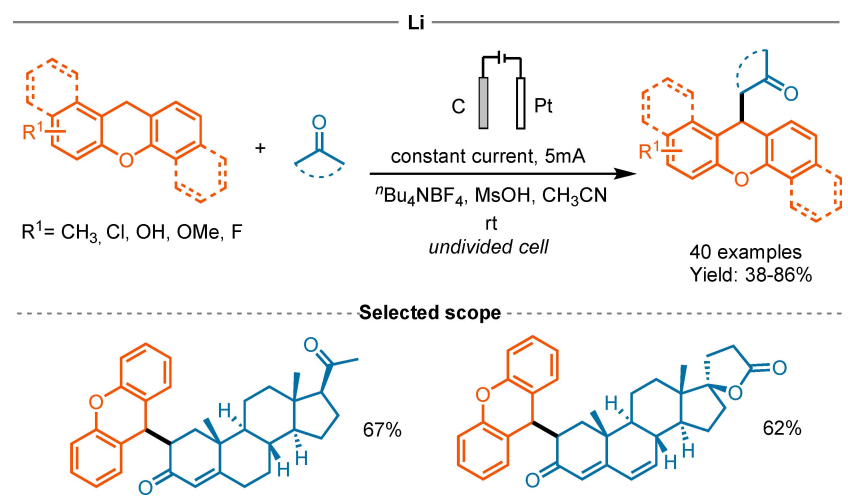

Scheme 27. Electrochemical dehydrogenative cross-coupling of xanthenes with ketones.

has also proven to be valid for anodic oxidation, as we will see later in this review. Generally, two subsequent single-electron oxidations lead to the formation of a stabilized carbocation that is able to react with a suitable nucleophile. In the present case, ketones were found trapping the reactive species generated at the anode through $\mathrm{C}-\mathrm{C}$ bond formation with the $\alpha$-carbon. Interestingly, the addition of quantitative amounts of $\mathrm{MsOH}$ was necessary for the reaction to proceed. If the reaction was conducted in its absence, solely the oxidation product xanthene-9-one was obtained. $\mathrm{MsOH}$ is believed to promote the enolization of the ketone, aiding the nucleophilic attack. The reaction proved to have good selectivity towards secondary $\mathrm{C}\left(s p^{3}\right)-\mathrm{H}$ adjacent to the carbonyl of ketones. This was particularly clear during the functionalization of progesterone and canrenone both characterised by the presence of other acidic $\mathrm{C}-\mathrm{H}$ bonds.

\section{2. $\mathrm{C}\left(s p^{3}\right)-\mathrm{O}$ Bond Formation}

The ability to oxidize selectively $\mathrm{C}-\mathrm{H}$ benzylic bonds combined with oxidizing reagents or oxygen nucleophiles opens a straightforward way to green and innovative $\mathrm{C}-\mathrm{O}$ bond formation. Nonetheless, as already mentioned, over-oxidation poses a major threat, thus hampering the applicability and the selectivity of direct electrolysis. Early work from Yoshida demonstrated the cationic pool method to be a valid solution. Benzylic cations were stabilized through the formation of alkoxysulfonium ions by reaction with dimethyl sulfoxide, to form either carbonyl compounds or benzyl alcohols, respectively by chemical oxidation ${ }^{[69 a]}$ or hydrolysis. ${ }^{[696]}$

In 2013, Little and collaborators employed an indirect electrolysis process for the oxygenation of benzylic methylene. ${ }^{[70]}$ They optimised the use of TEMPO as a redox catalyst in combination with catalytic amounts of bromide ions, exploiting electrolysis in the role of sacrificial oxidant. The trans- 
formation, conducted in a two-phase electrolytic medium (Scheme 28), starts in the aqueous phase with the oxidation of bromide anions to hypobromite at the anode. At the interphase, TEMPO engages an electron transfer with this reactive bromine species being oxidized to an oxammonium cation. Finally, the benzylic substrate is activated via single-electron oxidation followed by base promoted proton-transfer/ electron-transfer sequence. The newly formed cation is trapped by water to give the corresponding ketone. Indirect electrolysis allows for electrochemical processes at lower potentials increasing selectivity and avoiding side reactions. The reaction was mainly restricted to a benzylic position adjacent to a heteroatom as in the case of tetrahydroisoquinolines, isochromane and benzyl ethers. Nonetheless xanthene gave the corresponding xanthenone in excellent yields.

In the same year, Roth and collaborators ${ }^{[71]}$ explored the microfluidic electrochemical reaction conditions for the benzylic oxidation of tolyl-based substrates (Scheme 29). In this case, the researchers aimed to provide an upstream solution to overoxidation. Implementing continuous-flow technology in a constant current electrolysis process, they were able to modulate the electron equivalents (Faradays per mole, F/mol) provided to the substrate by simply adjusting the flow rate. This allowed the selective synthesis of either the four-electron or the six-electron products, respectively, while avoiding overoxidation. As a result, in the case of $p$-methoxytoluene, an increase in available electron equivalents, from $5 \mathrm{~F} / \mathrm{mol}$ to $8 \mathrm{~F} / \mathrm{mol}$, provided the

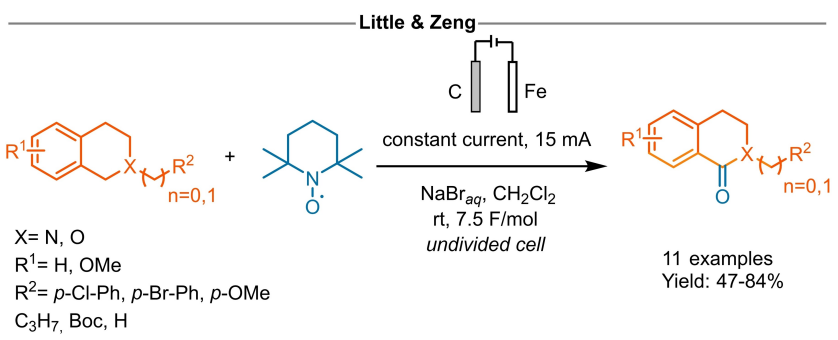

Scheme 28. TEMPO-mediator for indirect electrochemical oxidation.

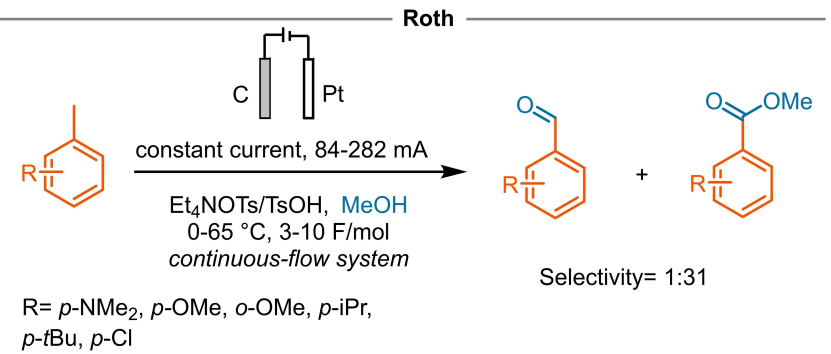

Scheme 29. Continuous-flow microfluidic electrochemical oxidation. methyl ester selectively. However, screening of methyl arenes bearing a variety of electron-donating and -withdrawing groups demonstrated the substitution pattern to play a major role in the reaction by strongly influencing the oxidation potential.

Similarly, Jensen and collaborators ${ }^{[72]}$ reported a scalable flow system for the benzylic radical generation and oxidation to ketone, employing air oxygen as co-oxidant while $N$-hydroxyphtalimide acts as mediator via HAT. Notably, reductive decomposition of NHPI was prevented by employing a cation-exchange membrane, allowing the use of catalytic amounts of phthalimide (Scheme 30).

Recently, Liu and collaborators reported the use of tert-butyl hydroperoxide for the direct activation of benzylic $\mathrm{C}-\mathrm{H}$ bonds via HAT by the tert-butyl peroxyl radical formed through anodic oxidation (Scheme 31 ). [73] The reaction conditions were effective for the oxidation of a wide variety of substrates, spanning from variously substituted tolyl analogues to heteroaryl derivatives, providing the corresponding ketones in modest-to-excellent yields.

In 2020, an innovative and interesting electrochemical oxidation-induced benzylic $\mathrm{C}\left(s p^{3}\right)-\mathrm{H}$ etherification has been developed by Wang and collabo-

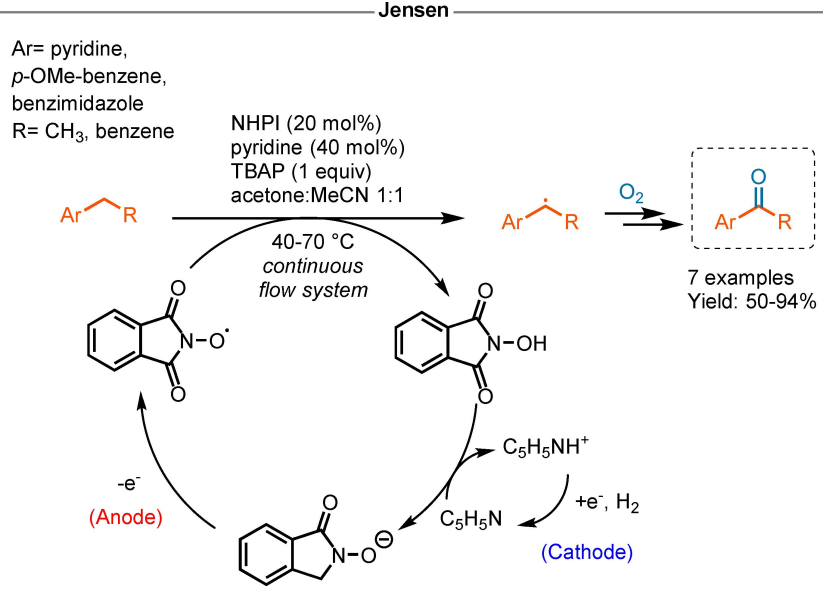

Scheme 30. Continuous-flow microfluidic electrochemical NHPI-mediated oxidation.

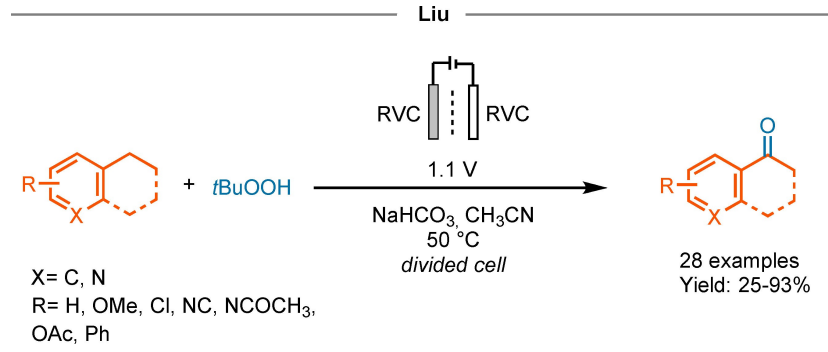

Scheme 31. Electrochemical benzylic oxidation tert-butyl peroxide mediated. 
rators (Scheme 32). ${ }^{[7]}$ Benzylic substrates were functionalised through anodic oxidation via base promoted $\mathrm{SET} /$ proton-transfer/SET sequence to form the carbocation which is subsequently trapped by an alcohol. A wide variety of functional groups on the alcohol side chain were tolerated, including unsaturated bonds, halogens, and ketone. Moreover, bulky tertiary alcohol, as tert-butanol and adamantanol could afford the desired product in moderate yields. Notably, the reaction did not require any redox mediator while still being able to yield high selectivity. The researchers suggest the disparity of redox potential between alcohols and benzyl $\mathrm{C}\left(s p^{3}\right)-\mathrm{H}$ compounds to be the reason behind this high selectivity.

\section{3. $\mathrm{C}\left(s \mathrm{p}^{3}\right)-\mathrm{N}$ Bond Formation}

Electrochemical direct $\mathrm{C}\left(s p^{3}\right)-\mathrm{N}$ bond formation appears as a streamline application as the anodic oxidation can be easily employed in the formation of carbon centred radicals and cations to be subsequently trapped by a N-based nucleophile. Nonetheless, few critical issues have hindered the development in this direction, the foremost being the overoxidation of the coupling product. Since the newly formed amines are usually characterized by redox potentials lower or comparable to the ones of the starting material, the product will start degrading before full conversion is reached. Secondly, the carbocation formed at the anode needs to be stable enough not to be trapped by a weak nucleophile (i.e. solvent or electrolytes), or form double bond via elimination. In this section, we will discuss the latest advances and strategies that led to successful coupling.

In 2014, Yoshida and collaborators ${ }^{[75]}$ reported a protocol for the electro-oxidative direct functionalization of a benzylic $\mathrm{C}\left(s p^{3}\right)-\mathrm{H}$ site with imidazoles (Scheme 33). Although metal- and organo-catalysed processes have been previously reported in chemically oxidative conditions, these suffered from overoxidation of the coupling product, requiring excess amount of benzylic starting material (up to 10 equiv.); thus hindering their applicability. This problem would be enhanced during the electrochemical oxidation. The solution lies in the formation of an imidazolium

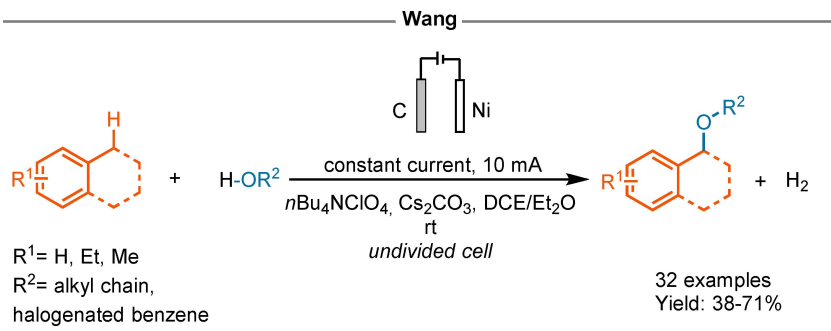

Scheme 32. Electrochemical oxidation-induced etherification.

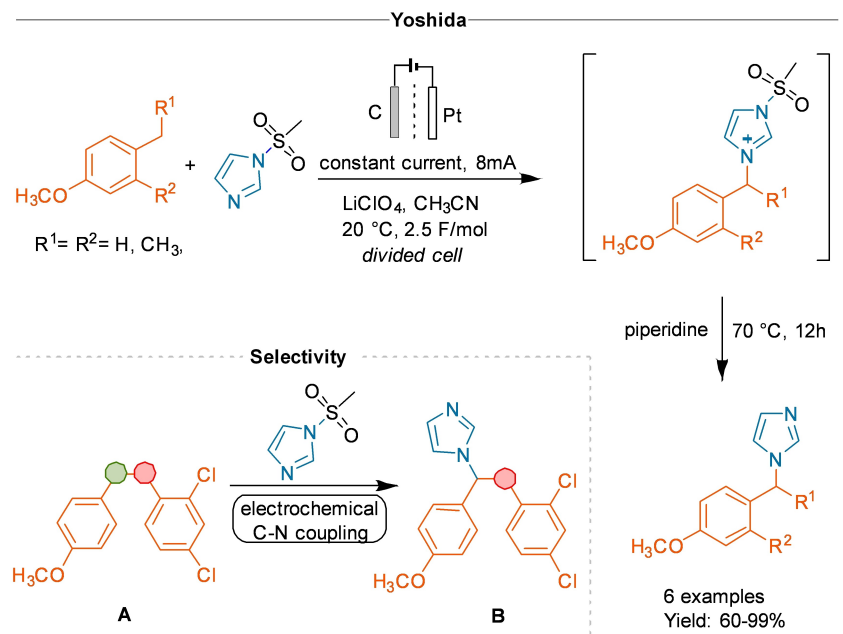

Scheme 33. Electro-oxidative direct functionalization of a benzylic $\mathrm{C}\left(s p^{3}\right)-\mathrm{H}$ site with imidazoles.

intermediate by using a protected imidazole that was not susceptible to oxidation at the same potential. Subsequent deprotection under non electrooxidative conditions delivered the desired coupling product. Out of the several protecting groups screened, mesyimidazole gave the best yields. Notably, the reaction proved to be selective for electron rich benzylic rings. The treatment of A (Scheme 33) gave selectively the product $\mathbf{B}$ by an exclusive attack on the electron rich benzylic position. The same selectivity was found for 3,4-dimethylmethoxybenzene where only the paramethyl was functionalized.

In 2017, the same group exploited a similar approach for the synthesis of protected benzylamines (Scheme 34). ${ }^{[76]} \mathrm{N}$-tosyldiphenylsulfilimine was employed as a coupling partner to form the corresponding benzylaminosulfonium ion. Treatment with tetrabuthylammonium iodide gave the desired protected amine. The reaction conditions tolerated a wide range of substituents on the benzylic ring and could also be

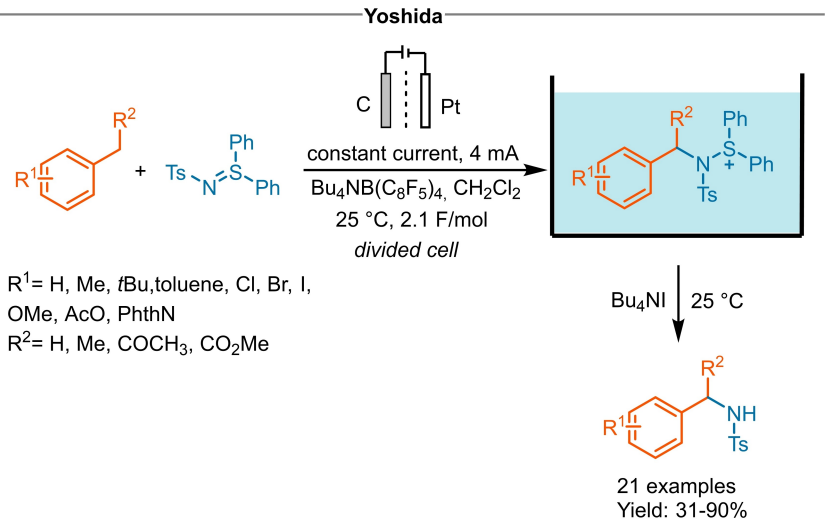

Scheme 34. Electrochemical benzylic $\mathrm{C}-\mathrm{H}$ amination. 
applied to a secondary benzylic position and methylheteroaromatics. The high redox potential of $\mathrm{N}$ tosyldiphenylsulfilimine allowed for the activation of benzylic substrates in a wide range of oxidation potential $(1.38-1.94 \mathrm{~V})$.

In 2018 , Zeng and collaborators ${ }^{[77]}$ reported the intermolecular cross dehydrogenative coupling between xanthenes and alkoxyamides (Scheme 35). The reaction, performed in an undivided cell at galvanostatic conditions, could be catalysed via indirect electrolysis by both bromide salts and ferrocene providing moderate to good yields. The reaction tolerated a variety of amides although strong electronic effects, both donating and withdrawing, hampered the reaction and lowered the yield ( $p$-OMe and $\left.p-\mathrm{NO}_{2}\right)$. In this work, the scope was limited to xanthene as in case of other substrates decomposition of the amide was observed. In 2019, two reports from $\mathrm{Li}^{[78]}$ and Wang ${ }^{[79]}$ further explored the electrochemical $\mathrm{C}-\mathrm{H}$ functionalization of xanthenes. In both cases direct electrolysis of xanthenes led to the formation of an activated intermediate, either as a cation or a radical, which was subsequently trapped with the formation of a $\mathrm{C}-\mathrm{N}$ bond. A wide variety of xanthene derivatives and azoles yielded the desired CDC products.

Wang and collaborators extended the scope to imides, which were found behaving differently as compared to azoles (Scheme 36). ${ }^{[79]}$ A divided cell experiment, where all the reagents were added in both chambers, gave traces or no product with imides, suggesting that the nucleophilic imidyl anion is formed

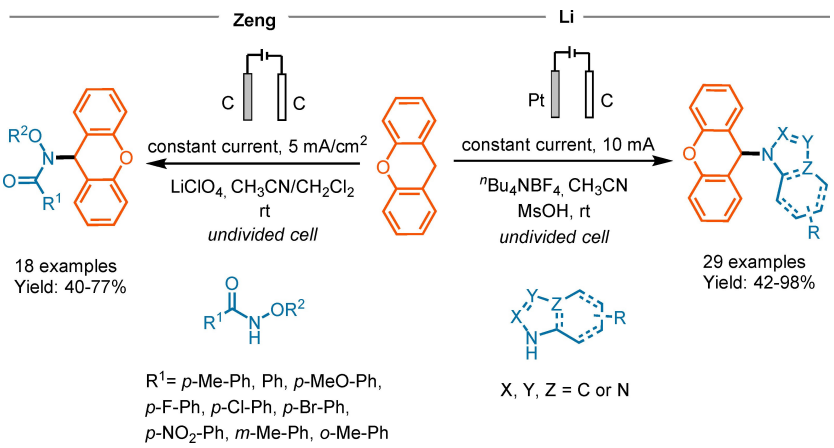

Scheme 35. Xanthenes electrochemical amination.

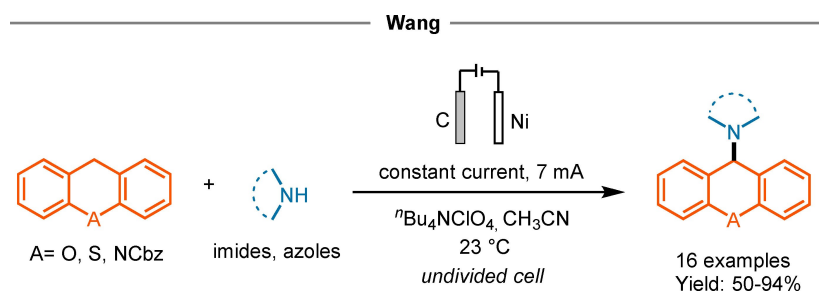

Scheme 36. Xanthenes electrochemical C-N bond formation. by either direct cathodic reduction or via deprotonation by basic species formed at the cathode.

It is worth mentioning that the benzotriazole oxidative $\mathrm{C}\left(s p^{3}\right)-\mathrm{H} / \mathrm{N}-\mathrm{H}$ cross-coupling was already reported by Lei ${ }^{[80]}$ in 2017 . Although the research was not specifically focused on benzyl $\mathrm{C}-\mathrm{H}$ functionalization, two examples were reported with isochromane and 2,4,6-trimethylphenol (Scheme 37).

Nitrogen based heterocycles are of great interest as they are widely represented in bioactive compounds. ${ }^{[81]}$ Muñiz and collaborators envisioned the application of electrochemical $\mathrm{C}\left(s p^{3}\right)-\mathrm{H} / \mathrm{N}-\mathrm{H}$ dehydrogenative coupling for the formation of cyclic amines through an intramolecular pathway. The assumption proved to be successful using phenyl-tethered tosylamides, and in 2018 they reported the synthesis of five and sixmembered amines via benzylic anodic oxidation to benzyl cation, followed by nucleophilic attack by sulphonamide ${ }^{[82]}$ (Scheme 38).

Clear evidence supporting the mechanism came from cyclovoltammetry (CV) studies as the lower oxidation potential allows for the selective oxidation of the benzyl position. Moreover, control studies using the electron-poor pyridine analogue, that failed at giving the desired product, showed that in this case oxidation of the sulphonamide happens first, hampering the overall reaction.

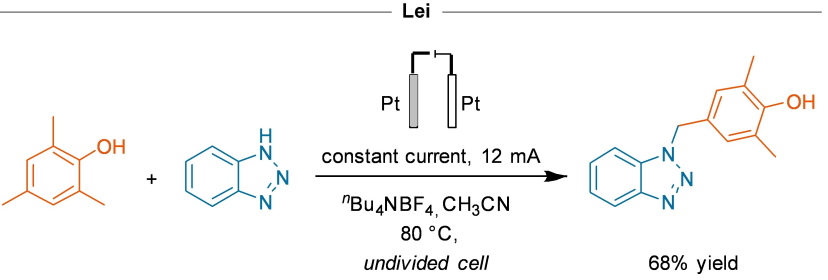

Scheme 37. 2,4,6-Trimethylphenol functionalization.

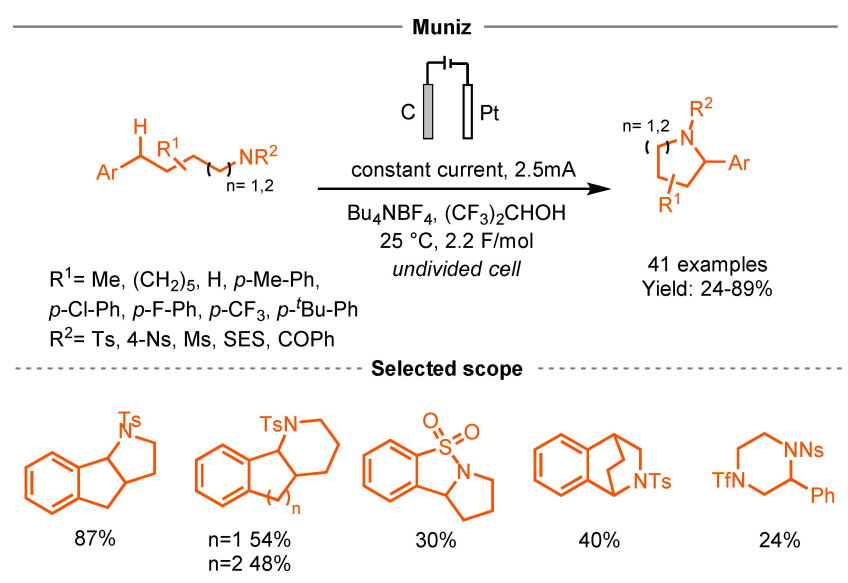

Scheme 38. Electrochemical dehydrogenative coupling to the formation of cyclic amines. 
The same year Wang and collaborators reported an electrocatalysed intermolecular synthesis of imidazofused N-heterocycles ${ }^{[83]}$ (Scheme 39). Methyl $N$-heteroaromatics reacted with benzylamines or $\alpha$-amino acids with the tandem formation of two $\mathrm{C}-\mathrm{N}$ bonds to give the cyclised imidazoquinoline. The reaction proceeds via indirect electrolysis mediated by iodine to form a benzyl-iodide intermediate. The latter was susceptible to nucleophilic attack from the aminated counterpart to which cyclization followed. In a control experiment, 2-(iodomethyl)quinoline was used as starting material in standard condition. Formation of the desired product in comparable yields supports the hypothesis of a halogenated intermediate.

Halo-assisted oxidation has proven to be a versatile strategy for electrochemical cross-coupling. ${ }^{[84]}$ In a work from Zeng's group ${ }^{[85]}$ (Scheme 40) this was found effective in the formation of $N$-centred amidyl radicals known for their peculiar electrophilicity. ${ }^{[86]}$ This activation has been applied to the intramolecular amination of both $\mathrm{C}\left(s p^{2}\right)-\mathrm{H}$ and $\mathrm{C}\left(s p^{3}\right)-\mathrm{H}$ bonds. In the case of benzylic functionalization, the authors suggest a 1,5-hydrogen atom transfer to be involved in

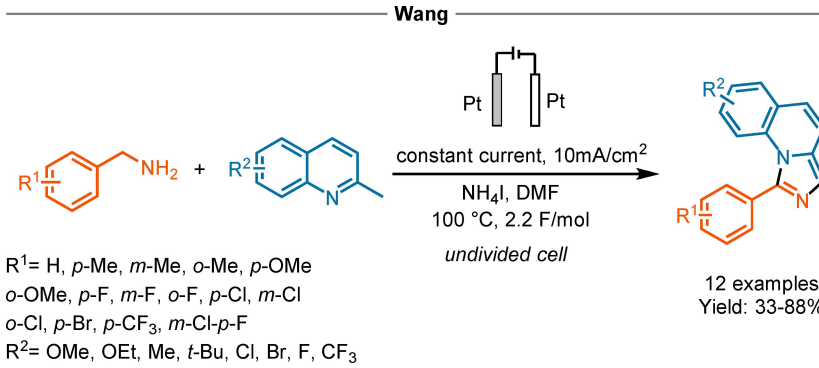

Scheme 39. Electrocatalysed intermolecular synthesis of imidazo-fused N-heterocycles.

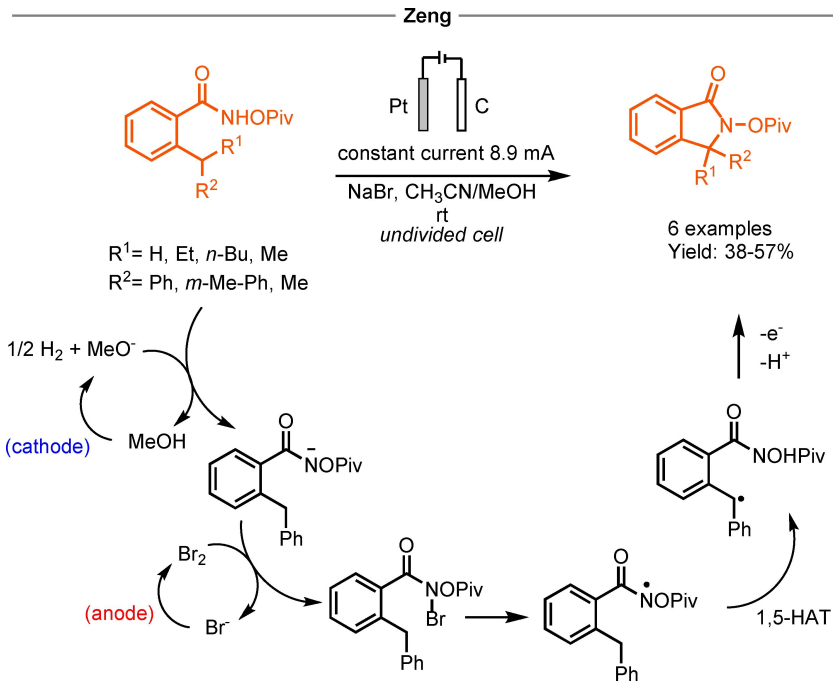

Scheme 40. Halo-assisted intramolecular amination. the formation of the benzyl radical which is susceptible of further oxidation and cyclization.

In 2018, Lei and collaborators reported the synthesis of variously substituted 2-phenyl pyrrolidines via electrocatalysis (Scheme 41). ${ }^{[87]}$ It was found that the reaction could proceed only in the presence of acetate anions. This can be explained by the coordination via hydrogen bond formation of the $\mathrm{NH}$, aiding the initial oxidation and deprotonation. The interaction was confirmed by a downfield shift in the proton NMR spectrum and a decrease of oxidation potential in the CV.

Flow chemistry is an efficient strategy to overcome the inherent instability of certain products to the reaction conditions. The immediate transfer of the newly formed molecules into a benign environment avoid side reactions and yield decrement. This concept has proven to be true in the case of benzyl acetamides electrochemical synthesis. In 2017, Ley and collaborators optimised the continuous production of substituted benzyl amides from alkyl aryls via anodic oxidation, to form a carbocation which was subsequently trapped by acetonitrile used as solvent (Scheme 42). ${ }^{[8]}$ This electrochemical version of the Ritter reaction was known to suffer from poor yield due to overoxidation. ${ }^{[89]}$ Continuous flow conditions, reducing the residence time of the product and allowing prompt quenching of the excess acid in the mixture, provided a valid solution.
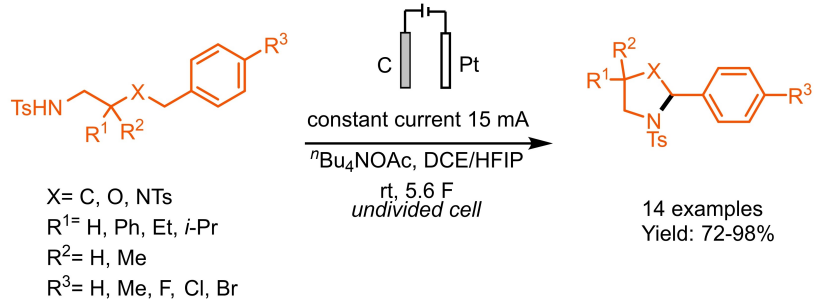

Scheme 41. Pyrrolidine synthesis via electrocatalysis.

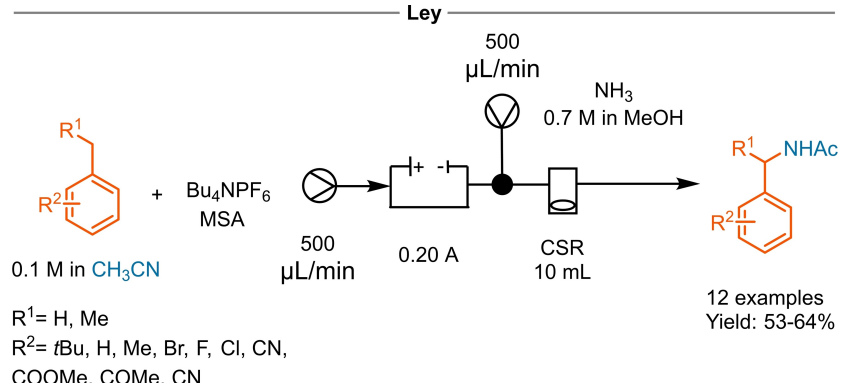

Scheme 42. Continuous-flow production of substituted benzyl amides. 


\section{4. $\mathrm{C}\left(s \mathrm{p}^{3}\right)-\mathrm{P}$ Bond Formation}

Electrochemical phosphonylation has attracted great interest in the past few years as it allows for a more sustainable approach. Avoiding the use of harsh conditions and highly reactive or sensitive reagents, electrochemical functionalization has found a fertile ground in the field of organophosphorus compounds. ${ }^{[90]}$ In 2019, Lei and collaborators reported a protocol for $\mathrm{C}\left(s p^{3}\right)-\mathrm{H}$ phosphonylation under exogenous-oxidantfree conditions via catalyst-free electrochemical oxidation (Scheme 43). ${ }^{[91]}$ Best yields were obtained with carbon rod anode and platinum plate cathode in an undivided cell using ${ }^{n} \mathrm{Bu}_{4} \mathrm{NPF}_{6}$ in acetonitrile. Xanthane and $N$-methyl-9,10-dihydroacridine gave the desired product in good yield. The reaction conditions were also applicable to an $N, N$ - dimethylaniline derivative and $N$-phenyl-tetrahydroisoquinoline although giving lower yields.

The same year Ding and collaborators optimised the electrochemical phosphonylation of unprotected benzyl secondary amines (Scheme 44 ) ${ }^{[92]}$ Interestingly, the addition of a Brønsted base was found to be crucial to get good yields. Sodium acetate, that gave the best results, is believed to coordinate with both the positive iminium ion generated by oxidation of benzylamine,

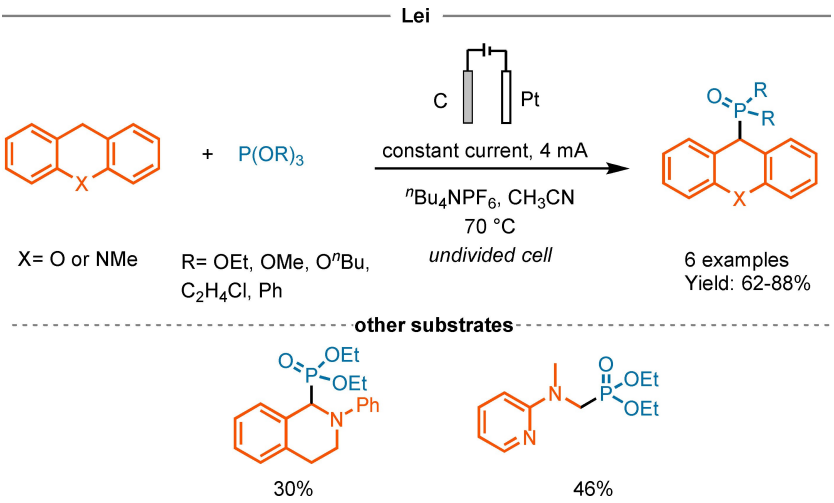

Scheme 43. $\mathrm{C}\left(s p^{3}\right)-\mathrm{H}$ phosphonylation via electrochemical oxidation.

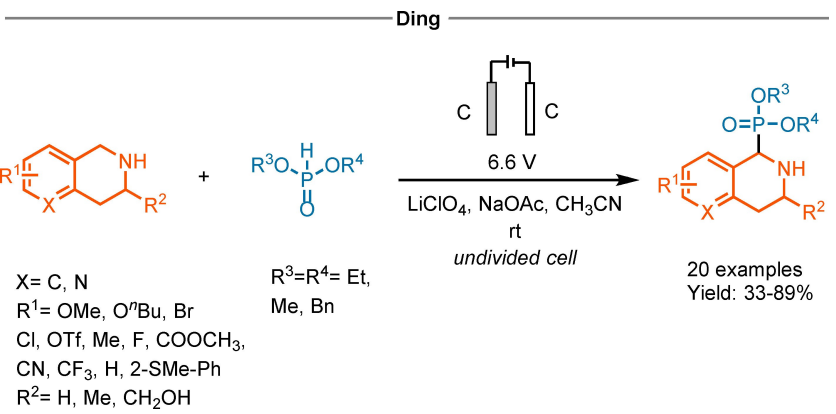

Scheme 44. Phosphonylation of unprotected benzyl secondary amines. and the phosphonate facilitating $\mathrm{C}-\mathrm{P}$ bond formation and abstracting one proton forming acetic acid. Subsequently, protons are reduced to dihydrogen at the cathode, with the regeneration of acetate anion.

In 2020, Leonel and collaborators have reported the phosphonylation of boc-protected tetrahydroisoquinoline (THIQ) with dimethyl phosphite (Scheme 45). ${ }^{[93]}$ The reaction did not require any additive and is believed to proceed via convergent paired electrolysis by anodic two-electron oxidation of THIQ- $N$-Boc and single electron reduction of the alkyl phosphite to the phosphite anion. This reactive species could then attack the iminium cation to give the desired product.

\subsection{C $\left(s p^{3}\right)-$ Halogen Bond Formation}

In previous sections, we have shown the versatility of halogen-based mediators for the electrochemical oxidative cross-coupling. Furthermore, halogenated compounds allow for efficient functionalization. An early report from Raju and collaborators demonstrated the applicability of electrochemical conditions for the direct $\mathrm{C}\left(s p^{3}\right)-\mathrm{H}$ bromination of alkylarenes (Scheme 46). ${ }^{[94]}$ The reaction was carried out in an undivided cell via two-phase electrolysis, using chloroform and aqueous sodium bromide solution $(60 \%)$ containing a catalytic amount of $\mathrm{HBr}$. Benzyl bromides were obtained in good to excellent yields.

In 2018, Stahl's group applied $N$-hydroxyphthalimide (NHPI) catalysis towards $\mathrm{C}-\mathrm{I}$ bond formation obtaining benzyl iodides in good yields

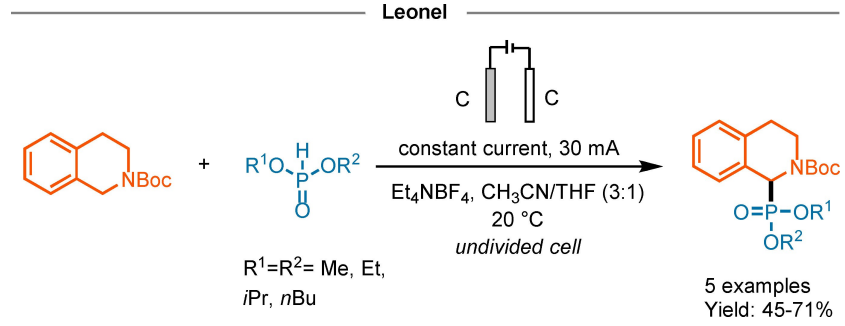

Scheme 45. Phosphonylation of boc-protected tetrahydroisoquinoline.

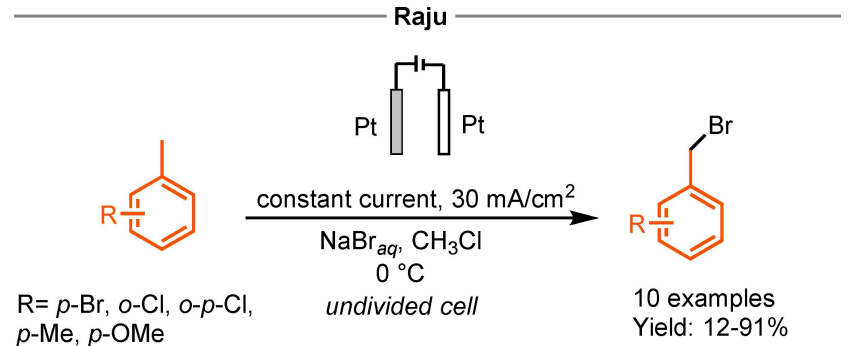

Scheme 46. Electrochemical direct $\mathrm{C}\left(s p^{3}\right)-\mathrm{H}$ bromination of alkylarenes. 
(Scheme 47). ${ }^{[95]}$ Moreover, the generation of halogenated products was applied to the synthesis of relevant bioactive compounds by sequential iodination/alkylation.

\section{Benzylic C(sp $\left.{ }^{3}\right)$-H Functionalization via Synthetic Photoelectrochemistry}

The previous sections gave a representative glance to the fast-developing and widespread application of photo-redox catalysis and electrochemistry to organic synthesis that characterized the past decade. These two enabling techniques appear as complementary tools in the accomplishment of redox-driven organic substrate functionalization, the first being highly specific and the second allowing for a virtually infinite range of redox potentials. Merging them in a single reaction sounds as a perfect match in combining the advantages and by overcoming specific week spots. Although, this field is still in its infancy, during the past couple of years several groups reviewed the latest reports underlining the increasing interest for the topic. ${ }^{[96]}$ For clarity, the nomenclature proposed by Barham and König will be adopted. $^{[96 \mathrm{~b}]}$

Electrochemical processes conducted using specific semiconductor electrodes benefit from an additional source of energy associated with the photoelectric effect. As first discovered by Becquerel in the 19th century, light irradiation can generate current in a conductive material. ${ }^{[97]}$ The development of photoelectrochemical cells (PEC) allowed chemists to exploit the high energy electron-hole pairs generated on the surface of the photoanode by incident photons. This has been exploited for the oxidation of high redox potential substrates, ${ }^{[98]}$ as in the widely explored water hydrolysis, ${ }^{[99]}$ and to keep low potentials in the system and therefore avoiding side reactions. ${ }^{[100]}$ The latter is the concept applied by Berlinguette and collaborators in their work from $2017,{ }^{[101]}$ where oxidation of organic substrates was obtained with $60 \%$ reduction in electrical energy compared to the classical electrochemis-

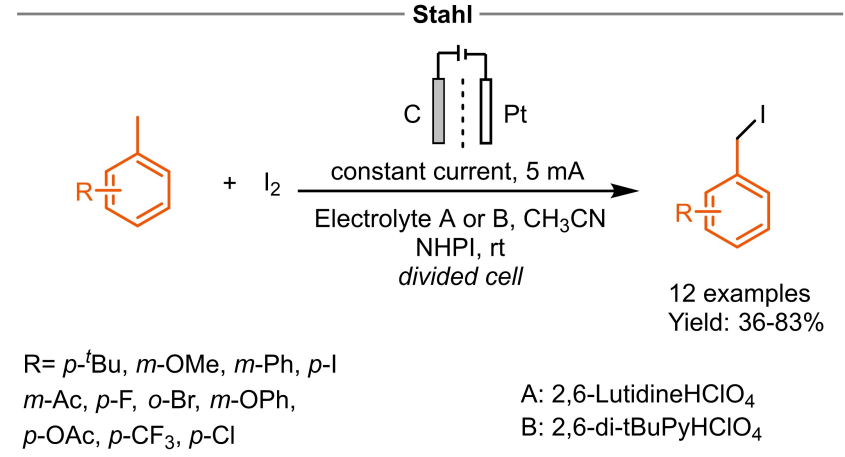

Scheme 47. Electrochemical C-I bond formation. try. They developed a protocol using $\mathrm{BiVO}_{4}$ photoanodes in acetonitrile, to avoid photo-corrosion, affording NHS-mediated $\mathrm{C}\left(s p^{3}\right)-\mathrm{H}$ oxidation of tetralin (Scheme 48). Furthermore, the effect of light irradiation was tangible while measuring the drop in oxidation potential of the NHS anion to the NHS radical of $1 \mathrm{~V}$ compared to standard electrochemistry.

In 2019 Wu's group applied similar conditions for the phosphorylation of $N$-aryl-tetrahydroisoquinilines with phosphine oxides or phosphite esters (Scheme 49). ${ }^{[102]}$ The reaction was carried out in acetonitrile using $\mathrm{BiVO}_{4}$ as working electrode and $\mathrm{Pt}$ plate as counter electrode with a cell potential of $0.5 \mathrm{~V}$ vs $\mathrm{Ag} / \mathrm{AgCl}$ in an undivided cell with blue LED irradiation. Addition of 0.2 equiv. $N$-hydroxyphthalimide as mediator caused a tremendous increase in yield, from 39\% to 93\%. Moreover, the augmented photocurrent caused a decrease in the potential required to produce $5.0 \mathrm{~mA}$ down to $0.1 \mathrm{~V}$. Compared to electrochemical conditions, using a reticulated vitreous carbon anode and a $\mathrm{Pt}$ plate cathode, more than $90 \%$ of the external bias input was saved by PEC conditions obtaining comparable or higher yields.

Zhou and collaborators reported the oxidation of 3methylpyridine to the corresponding acid via PEC synthesis (Scheme 50). ${ }^{[103]}$ Their setup consisted in a dual-chamber cell equipped with a $\mathrm{WO}_{3}$ film photoanode, a $\mathrm{Pt}$ wire cathode and a proton exchange membrane.

A different approach to synthetic photoelectrochemistry is represented by reactions where light irradiation directly activates reagents or intermediates.

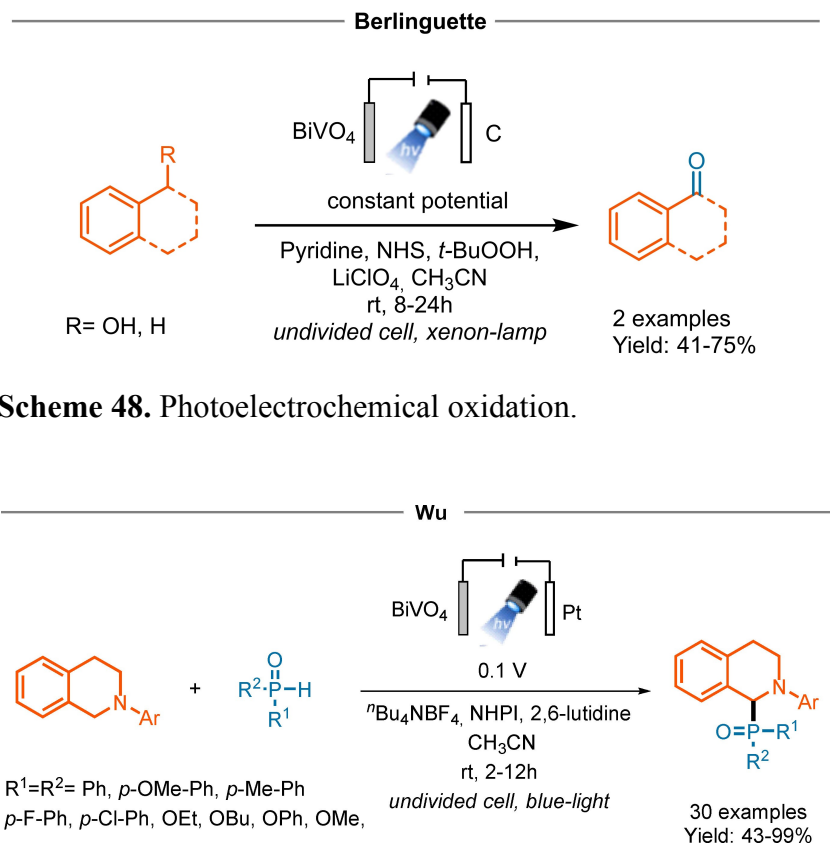

Scheme 49. Benzylic C $\left(s p^{3}\right)-\mathrm{H}$ phosphorylation via PEC. 


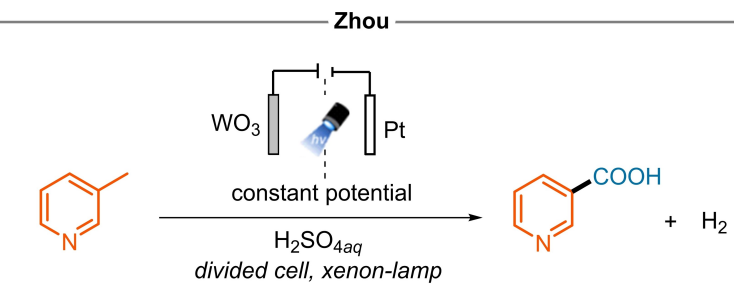

Scheme 50. Methylpyridine PEC oxidation.

In 2019, Stahl's group merged iodide-mediated electrochemistry and photo-induced homolytic cleavage of $\mathrm{N}-\mathrm{I}$ bonds in a Hofmann-Löffler-Freytag-type amination of $\mathrm{C}\left(s p^{3}\right)-\mathrm{H}$ bonds. ${ }^{[104]}$ The model reaction is the cyclization of $N$-alkyl sulfonamide derivatives to pyrrolidine (Scheme 51). Initial anodic oxidation of $\mathrm{I}^{-}$ to molecular iodide with concomitant production of hydrogen and Brønsted base at the cathode starts the reaction. Base-promoted iodination of the $\mathrm{N}-\mathrm{H}$ substrate generates the photoactive intermediate that undergoes homolysis to form a $N$-centred radical. Subsequent 1,5-HAT forms the benzylic radical which trapped by $\mathrm{I}_{2}$ gives a benzylic iodide. The latter is susceptible to nucleophilic attack by the nitrogen. This

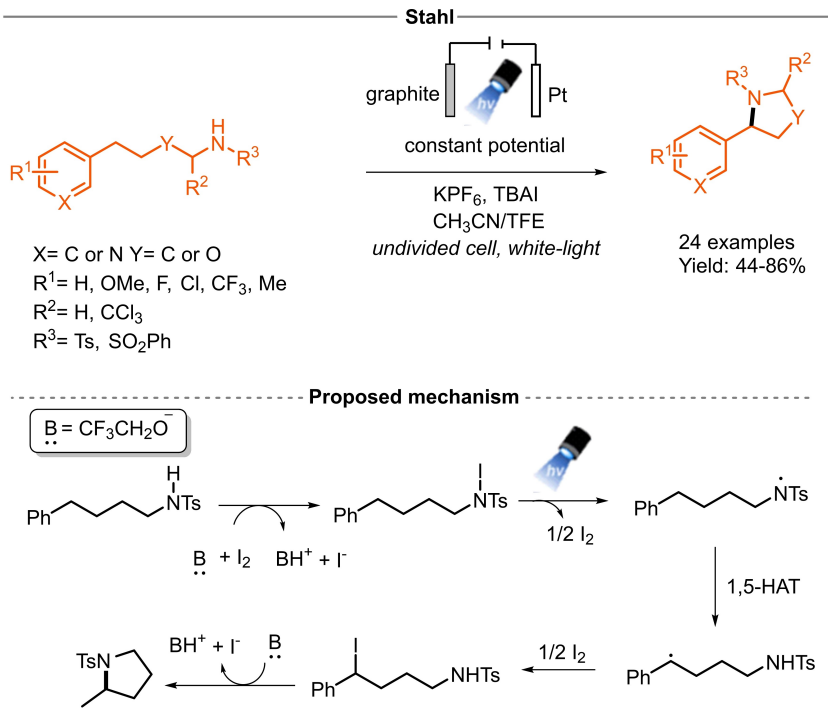

Scheme 51. Intramolecular $\mathrm{C}\left(s p^{3}\right)-\mathrm{N}$ bond formation.

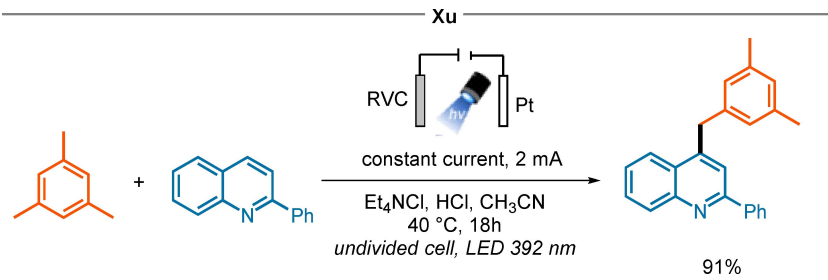

Scheme 52. Photoelectrochemical dehydrogenative cross-coupling. is an example of decoupled photoelectrochemistry as light irradiation and the electrochemical component are involved in independent mechanistic steps. When imidates were used instead, oxazolines could be efficiently synthetized.

In 2020, Xu's group reported an example of photoelectrochemical dehydrogenative cross-coupling between alkyl arene and phenyl-quinoline (Scheme 52). ${ }^{[105]}$ The reaction involves intimate cooperation between photochemical and electrochemical components leading to the generation of the active species. Firstly, molecular chlorine is generated from chloride anions through anodic oxidation. Subsequently, $\mathrm{Cl}_{2}$ undergoes photochemical homolysis to a chlorine radical able to abstract a hydrogen from an aliphatic carbon. The alkyl radical is finally trapped by a heteroarene.

Finally, there is a recently published work from Lei's group regarding $\mathrm{C}\left(s p^{3}\right)-\mathrm{H}$ bonds azidation (Scheme 53). ${ }^{[106]}$ Their approach has lifted manganesecatalysed oxidative azidation by replacing stoichiometric amounts of external oxidants under photoelectrochemical conditions. The photocatalyst, upon excitation, activates the $\mathrm{C}\left(s p^{3}\right)-\mathrm{H}$ bond via HAT. Anodic

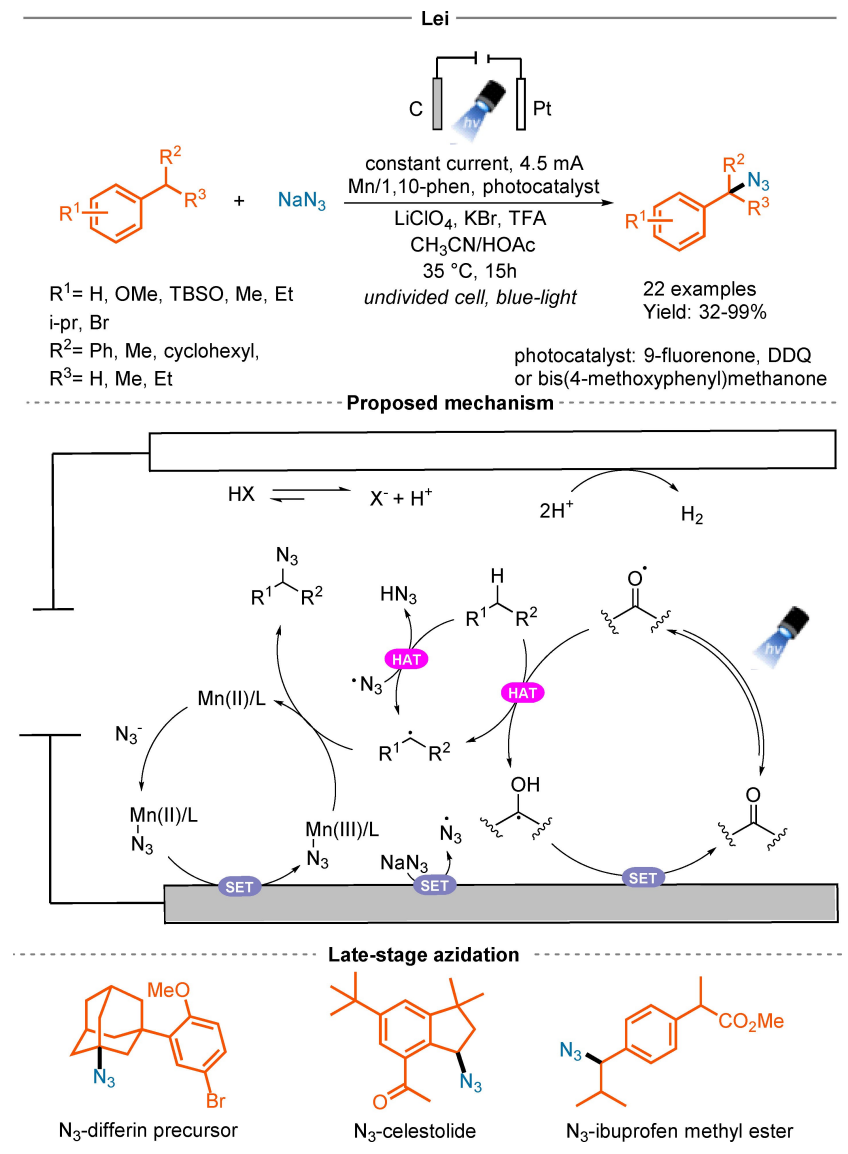

Scheme 53. Benzylic $\mathrm{C}\left(s p^{3}\right)-\mathrm{H}$ azidation by manganese mediated photoelectrochemistry. 
oxidation is involved in the generation of azide radicals from $\mathrm{NaN}_{3}$, oxidation of the $\mathrm{Mn}(\mathrm{II}) / \mathrm{L}-\mathrm{N}_{3}$ intermediate to $\mathrm{Mn}(\mathrm{III}) / \mathrm{L}-\mathrm{N}_{3}$ and the regeneration of the photocatalyst. The reaction conditions successfully delivered the benzylic azidation of a variety of substrates in moderate to good yields. Noticeably, tertiary alkyl groups were able to efficiently undergo azidation. Late-stage azidation of bioactive compounds further demonstrated the applicability of this innovative protocol.

\section{Conclusions and Outlook}

The functionalization of the benzylic position is a strategic step for the synthesis of complex molecular structures and various bioactive molecules. Intrinsic reactivity challenges generated wide interest towards effective and selective synthetic pathways. In this review we gave a representative description of the impact that photo- and electrochemistry had in this field, showing the benefits of engaging direct activation of the benzylic position through the generation of radical species in a safer way, thus reducing the number of synthetic steps and allowing milder reaction conditions to access desired structural entities. Nonetheless, we believe it to be just a glance of the potential these enabling techniques have in revolutionizing organic synthesis. Throughout our examination, we encountered a variety of activation approaches by the means of HAT, organic catalysts, co-catalytic systems, and different types of energy transfer to face substrate related issues. We hope such comprehensive approach will help recognising reactivity patterns and guide the choice for tailored catalytic systems to further explore this prolific field. We also aim to drive attention to specific gaps that still need to be filled. More concentrated efforts need to be done in the field of benzylic activations, especially with regard to alcoholic functionalization. Visible-light mediated reactions and optimization of procedures for flow electrochemistry especially as a possible solution to overoxidation, not always avoidable by means of redox mediators. Great improvements have been done in recent years toward the standardization of these techniques that for long time relied on poorly reproducible home-made set-ups. Since powerful and accurate light sources as well as bench-top electrochemistry devices are now more easily available, we envision photo- and electrochemistry to spread even further and hope they will be fully integrated in every synthetic chemist's toolbox.

\section{Acknowledgements}

The authors wish to thank the FWO - Fund for Scientific Research-Flanders (Belgium) and the Research Fund of the University of Leuven (KU Leuven) for the financial support.
Monica Oliva is grateful to FWO for the PhD fellowship (FWO aspirant, grant number $11 F 4320 \mathrm{~N}$ ). This paper has been supported by the RUDN University Strategic Academic Leadership Program.

\section{References}

[1] a) D. C. Blakemore, L. Castro, I. Churcher, D. C. Rees, A. W. Thomas, D. M. Wilson, A. Wood, Nat. Chem. 2018, 10, 383-394; b) S. Govaerts, A. Nyuchev, T. Noel, J. Flow Chem. 2020, 10, 13-71; c) For a more detailed description of $\mathrm{C}-\mathrm{H}$ bonds energies and activation see: X. Xue, P. Ji, B. Zhou, J. Cheng, Chem. Rev. 2017, 117, 8622-8648.

[2] T. Cernak, K. D. Dykstra, S. Tyagarajan, P. Vachal, S. W. Krska, Chem. Soc. Rev. 2016, 45, 546-576.

[3] a) K. Godula, D. Sames, Science 2006, 312, 67-72; b) J. Wencel-Delord, F. Glorius, Nat. Chem. 2013, 5, 369-375; c) T. Cernak, K. D. Dykstra, S. Tyagarajan, P. Vachal, S. W. Krska, Chem. Soc. Rev. 2016, 45, 546576; d) M. Moira, J. J. Danona, T. A. Reekieb, M. Kassiou, Expert Opin. Drug Discovery 2019, 14, 11371149; e) W.-H. Rao, B.-F. Shi, Org. Chem. Front. 2016, 3, 1028-1047; f) Y.-H. Liu, Y.-N. Xia, B.-F. Shi, Chin. J. Chem. 2020, 38, 635-662.

[4] a) O. Baudoin, Chem. Soc. Rev. 2011, 40, 4902-4911; b) Y. Park, Y. Kim, S. Chang, Chem. Rev. 2017, 117, 9247-9301; c) Z. Chen, M.-Y. Rong, J. Nie, X.-F. Zhu, B.-F. Shi, J.-A. Ma, Chem. Soc. Rev. 2019, 48, 49214942; d) Q. Zhang, B.-F. Shi, Chin. J. Chem. 2019, 37, 647-656; e) S. Rej, Y. Ano, N. Chatani, Chem. Rev. 2020, 120, 1788-1887.

[5] P. E. M. Siegbahn, J. Phys. Chem. 1995, 99, 12723 12729.

[6] W. D. Jones, F. J. Feher, Acc. Chem. Res. 1989, 22, 91100.

[7] a) X. X. Guo, D. W. Gu, Z. Wu, W. Zhang, Chem. Rev. 2015, 115, 1622-1651; b) C. L. Sun, B. J. Li, Z. J. Shi, Chem. Rev. 2011, 111, 1293-1314.

[8] Hydrogen-Tranfer Reactions (Eds.: J. T. Hynes, J. P. Klinman, H.-H. Limbach, R. L. Schowen), Wiley-VCH Verlag GmbH \& Co. KGaA, 2007.

[9] Encyclopedia of Radicals in Chemistry, Biology and Materials (Eds.: C. Chatgilialoglu, A. Studer), John Wiley \& Sons, Ltd., 2012.

[10] M. Salamone, M. Bietti, Acc. Chem. Res. 2015, 48, 2895-2903.

[11] L. Capaldo, D. Ravelli, Eur. J. Org. Chem. 2017, 15, 2056-2071.

[12] C. B. Tripathi, T. Ohtani, M. T. Corbett, T. Ooi, Chem. Sci. 2017, 8, 5622-5627.

[13] D. Ravelli, S. Protti, M. Fagnoni, Acc. Chem. Res. 2016, 49, 2232-2242.

[14] a) K. Qvortrup, D. A. Rankic, D. W. C. MacMillan, J. Am. Chem. Soc. 2014, 136, 626-629; b) D. Hager, D. W. C. MacMillan, J. Am. Chem. Soc. 2014, 136, 16986-16989; c) J. D. Cuthbertson, D. W. C. MacMillan, Nature 2015, 519, 74-77; d) J. Jin, D. W. C. 
MacMillan, Nature 2015, 525, 87-90; e) Y. Y. Loh, K. Nagao, A. J. Hoover, D. Hesk, N. R. Rivera, S. L. Colletti, I. W. Davies, D. W. C. MacMillan, Science 2017, 358, 1182-1187; f) S. Kato, Y. Saga, M. Kojima, H. Fuse, S. Matsunaga, A. Fukatsu, M. Kondo, S. Masaoka, M. Kanai, J. Am. Chem. Soc. 2017, 139, 2204-2207.

[15] S. Feng, X. Xie, W. Zhang, L. Liu, Z. Zhong, D. Xu, X. She, Org. Lett. 2016, 18, 3846-3849.

[16] Z. Zuo, D. T. Ahneman, L. Chu, J. A. Terrett, A. G. Doyle, D. W. C. MacMillan, Science 2014, 345, 437440.

[17] D. R. Heitz, J. C. Tellis, G. A. Molander, J. Am. Chem. Soc. 2016, 138, 12715-12718.

[18] A. Albini, V. Dichiarante, Photochem. Photobiol. Sci. 2009, 8, 248-254.

[19] C. Xiaokai, L. Huangzhe, L. Zhan, Nat. Commun. 2019, 10,3549 .

[20] T. Kawasaki, N. Ishida, M. Murakami, J. Am. Chem. Soc. 2020, 142, 3366-3370.

[21] H.-P. Deng, Q. Zhou, J. Wu, Angew. Chem. Int. Ed. 2018, 130, 12843-12847.

[22] A. Dewanji, P. E. Krach, M. Rueping, Angew. Chem. Int. Ed. 2019, 58, 3566-3570.

[23] Y. Li, M. Lei, L. Gong, Nat. Can. 2019, 2, 1016-1026.

[24] N. Ishida, Y. Masuda, Y. Imamura, K. Yamazaki, M. Murakami, J. Am. Chem. Soc. 2019, 141, 19611-19615.

[25] H. Tanaka, K. Sakai, A. Kawamura, K. Oisaki, M. Kanai, Chem. Commun. 2018, 54, 3215-3218.

[26] a) Y. Zhang, W. Schilling, S. Das, ChemSusChem 2019, 12, 2898-2910; b) L. Revathi, L. Ravindar, W. Y. Fang, K. P. Rakesh, H. L. Qin, Adv. Synth. Catal. 2018, 360 , 4652-4698; c) A. Savateev, M. Antonietti, ACS Catal. 2018, 8, 9790-9808.

[27] J. Otera, Esterification: Methods, Reactions, and Applications, Wiley-VCH, Weinheim, 2003.

[28] a) T. Punniyamurthy, S. Velusamy, J. Iqbal, Chem. Rev. 2005, 105, 2329-2364; b) F. Recupero, C. Punta, Chem. Rev. 2007, 107, 3800-3842.

[29] G.-J. ten Brink, I. W. C. E. Arends, R. A. Sheldon, Science 2000, 287, 1636-1639.

[30] H. C. Ooi, H. Suschitzky, J. Chem. Soc. Perkin Trans. 1 1982, 2871-2875.

[31] a) E. Alcalde, M. Gisbert, J. M. Pons, L. Perez-Garcia, Tetrahedron 1996, 52, 15197-15208; b) J. Mlochowski, H. Wójtowicz-Mlochowski, Molecules 2015, 20, 10205-10243.

[32] a) Y. Bonvin, E. Callens, I. Larrosa, D. A. Henderson, J. Oldham, A. J. Burton, A. G. M. Barrett, Org. Lett. 2005, 7, 4549-4552; b) A. J. Catino, J. M. Nichols, H. Choi, S. Gottipamila, M. P. Doyle, Org. Lett. 2005, 7, 5167-5170; c) D. Zhang, C. Pan, Catal. Commun. 2012, 20, 41-45; d) D. Shen, C. Miao, S. Wang, C. Xia, W. Sun, Org. Lett. 2014, 16, 1108-1111; e) B. B. Wentzel, M. P. J. Donners, P. L. Alsters, M. C. Feiters, R. J. M. Nolte, Tetrahedron 2000, 56, 7797-7803; f) Y. Xie, Y. Yang, L. Huang, X. Zhang, Y. Zhang, Org. Lett. 2012, 14, 1238-1241.
[33] a) F.-T. Du, J.-X. Ji, Chem. Sci. 2012, 3, 460-465; b) Q. Lu, J. Zhang, G. Zhao, Y. Qi, H. Wang, A. Lei, J. Am. Chem. Soc. 2013, 135, 11481-11484; c) K. K. Toh, A. Biswas, Y. F. Wang, Y. Y. Tan, S. Chiba, J. Am. Chem. Soc. 2014, 136, 6011-6020; d) Y. Yan, P. Feng, Q. Z. Zheng, Y. F. Liang, J. F. Lu, Y. Cui, N. Jiao, Angew. Chem. Int. Ed. 2013, 52, 5827-5831; Angew. Chem. 2013, 125, 5939-5943; e) G. Zhang, L. Yang, Y. Wang, Y. Xie, H. Huang, J. Am. Chem. Soc. 2013, 135 , 8850-8853.

[34] H. Yi, C. Bian, X. Hu, L. Niu, A. Lei, Chem. Commun. 2015, 51, 14046-14049.

[35] X. Liu, L. Lin, X. Ye, C.-H. Tan, Z. Jiang, Asian J. Org. Chem. 2017, 6, 422-425.

[36] G. Pandey, S. Pal, R. Laha, Angew. Chem. Int. Ed. 2013, 52, 5146-5149; Angew. Chem. 2013, 125, 52505253.

[37] Y. Zhao, C. Zhang, K. F. Chin, O. Pytela, G. Wei, H. Liu, F. Bureš, Z. Jiang, $R S C A d v$. 2014, 4, 3006230067.

[38] a) F. Rusch, J.-C. Schober, M. Brasholz, ChemCatChem 2016, 8, 2881-2884; b) D. Pan, Y. Wang, M. Li, X. Hu, N. Sun, L. Jin, B. Hu, Z. Shen, Synlett 2019, 30, 218 224; c) L. C. Finney, L. J. Mitchell, C. J. Moody, Green Chem. 2018, 20, 2242-2249.

[39] W. Zhang, J. Gacss, I. W. C. E. Arends, F. Hollmann, Chem CatChem 2017, 9, 3821-3826.

[40] K. C. C. Aganda, B. Hong, A. Lee, Adv. Synth. Catal. 2019, 361, 1124-1129.

[41] D. Sarma, B. Majumdar, T. K. Sarma, ACS Sustainable Chem. Eng. 2018, 6, 16573-16585.

[42] L. Ren, M.-M. Yang, C.-H. Tung, L.-Z. Wu, H. Cong, ACS Catal. 2017, 7, 8164-8138.

[43] N. Serpone, A. V. Emeline, J. Phys. Chem. Lett. 2012, 3, 673-677.

[44] L.-Y. Jiang, J.-J. Ming, L.-Y. Wang, Y.-Y. Jiang, L.-H. Ren, Z.-C. Wang, W.-C. Cheng, Green Chem. 2020, 22, $1156-1163$.

[45] R. C. Betori, C. M. May, K. A. Scheidt, Angew. Chem. Int. Ed. 2019, 58, 16490-16494.

[46] a) F. R. Bisogno, M. Llpez-Vidal, G. de Gonzalo, $A d v$. Synth. Catal. 2017, 359, 2026-2049; b) J. H. Schrittwieser, S. Velikogne, M. Hall, W. Kroutil, Chem. Rev. 2018, 118, 270-348; c) R. A. Sheldon, D. Brady, M. L. Bode, Chem. Sci. 2020, 11, 2587-2605.

[47] L. Zhang, H. Yi, J. Wang, A. Lei, Green Chem. 2016 , $18,5122-5126$

[48] B. J. Lee, K. S. DeGlopper, T. P. Yoon, Angew. Chem. Int. Ed. 2020, 59, 197-202.

[49] G. Pandey, R. Laha, P. K. Mondal, Chem. Commun. 2019, 55, 9689-9692.

[50] a) H. M. L. Davies, J. R. Manning, Nature 2008, 451, 417-424; b) F. Collet, R. H. Dodd, P. Dauban, Chem. Commun. 2009, 34, 5061-5074; c) O. V. Zatolochnaya V. Gevorgyan, Nat. Chem. 2014, 6, 661-663.

[51] a) H. Lebel, K. Huard, S. Lectard, J. Am. Chem. Soc. 2005, 127, 14198-14199; b) K. Huard, H. Lebel, Chem. 
Eur. J. 2008, 14, 6222-6230; c) R. P. Reddy, H. M. L. Davies, Org. Lett. 2006, 8, 5013-5016.

[52] a) Y. Cheng, W. Dong, L. Wang, K. Parthasarathy, C. Bolm, Org. Lett. 2014, 16, 2000-2002; b) X. Zhang, M. Wang, P. Li, L. Wang, Chem. Commun. 2014, 50, 8006-8009; c) F. Jia, Z. Li, Org. Chem. Front. 2014, 1 , 194-214.

[53] G. Pandey, R. Laha, Angew. Chem. Int. Ed. 2015, 54, 14875-15092; Angew. Chem. 2015, 127, 15088-15092.

[54] D. M. Yan, Q. Q. Zhao, L. Rao, J. R. Chen, W. J. Xiao, Chem. Eur. J. 2018, 24, 16895-16901.

[55] X. Huang, J. T. Groves, ACS Catal. 2016, 6, 751-759.

[56] P. T. G. Rabet, G. Fumagalli, S. Boyd, M. F. Greaney, Org. Lett. 2016, 18, 1646-1649.

[57] G. Fumagalli, P. T. Rabet, S. Boyd, M. F. Greaney, Angew. Chem. Int. Ed. 2015, 54, 11481-11484; Angew. Chem. 2015, 127, 11643-11646.

[58] C. Hansch, A. Leo, S. H. Unger, K. H. Kim, D. Nikaitani, E. J. Lien, J. Med. Chem. 1973, 16, 12071216.

[59] C. Chen, K.-H. Xu, B. Yang, F.-L. Qing, Org. Lett. 2014, 16, 3372-3375.

[60] W. Xu, W. Wang, T. Liu, J. Xie, C. Zhu, Nat. Commun. 2019, 10, 1-8.

[61] C. W. Kee, K. M. Chan, M. W. Wong, C.-H. Tan, Asian J. Org. Chem. 2014, 3, 536-544.

[62] M. Xiang, Z.-K. Xin, B. Chen, C.-H. Tung, L.-Z. Wu, Org. Lett. 2017, 19, 3009-3012.

[63] a) J. Yoshida, K. Kataoka, R. Horcajada, A. Nagaki, Chem. Rev. 2008, 108, 2265-2299; b) Q. Yang, P. Fang, T. Mei, Chin. J. Chem. 2018, 36, 338-352.

[64] a) J. Yoshida, S. Suga, S. Suzuki, N. Kinomura, A. Yamamoto, K. Fujiwara, J. Am. Chem. Soc. 1999, 121 , 9546-9549; b) S. Suga, S. Suzuki, A. Yamamoto, J Yoshida, J. Am. Chem. Soc. 2000, 122, 10244-10245.

[65] R. Hayashi, A. Shimizu, J. Yoshida, J. Am. Chem. Soc. 2016, 138, 8400-8403.

[66] N. Fu, L. Li, Q. Yang, S. Luo, Org. Lett. 2017, 19, $2122-2125$

[67] Y.-Z. Yang, Y.-C. Wu, R.-J. Song, J.-H. Li, Chem. Commun. 2020, 56, 7585-7588.

[68] a) H. Choe, M. Farzan, Y. Sun, N. Sullivan, B. Rollins, P. D. Ponath, L. Wu, C. R. Mackay, G. LaRosa, W. Newman, N. Gerard, C. Gerard, J. Sodroski, Cell 1996, 85, 1135-1148; b) C. Sha, J. Han, F. Zhao, X. Shao, H. Yang, L. Wang, F. Yu, W. Liu, Y. Li, J. Pharm. Biomed. Anal. 2017, 146, 24-28.

[69] a) Y. Ashikari, T. Nokami, J. Yoshida, J. Am. Chem. Soc. 2011, 133, 11840-11843; b) Y. Ashikari, T. Nokami, J. Yoshida, Org. Lett. 2012, 14, 938-941.

[70] C. Li, C.-C. Zeng, L.-M. Hu, F.-L. Yang, S. J. Yoo, R. D. Little, Electrochim. Acta 2013, 114, 560-566.

[71] G. P. Roth, R. Stalder, T. R. Long, D. R. Sauer, S. W. Djurie, J. Flow Chem. 2013, 3, 34-40.

[72] Y. Mo, K. F. Jensen, Chem. Eur. J. 2018, 24, 10260 10265.

[73] J. A. Marko, A. Durgham, S. L. Bretz, W. Liu, Chem. Commun. 2019, 55, 937-940.
[74] H. Wang, K. Liang, W. Xiong, S. Samanta, W. Li, A. Lei, Sci. $A d v$. 2020, 6, eaaz0590.

[75] T. Morofuji, A. Shimizu, J. Yoshida, J. Am. Chem. Soc 2014, 136, 4496-4499.

[76] R. Hayashi, A. Shimizu, Y. Song, Y. Ashikari, T Nokami, J. Yoshida, Chem. Eur. J. 2017, 23, 61-64.

[77] M.-Y. Lin, K. Xu, Y.-Y. Jiang, Y.-G. Liu, B.-G. Sun, C.C. Zeng, Adv. Synth. Catal. 2018, 360, 1665-1672.

[78] Y.-Z. Yang, R.-J. Song, J.-H. Li, Org. Lett. 2019, 21, 3228-3231.

[79] X. Shao, L. Tian, Y. Wang, Eur. J. Org. Chem. 2019, 4089-4094.

[80] J. Wu, Y. Zhou, Y. Zhou, C.-W. Chiang, A. Lei, ACS Catal. 2017, 7, 8320-8323.

[81] a) R. D. Taylor, M. MacCoss, A. D. G. Lawson, J. Med. Chem. 2014, 57, 5845-5859; b) E. Vitaku, D. T. Smith, J. T. Njardarson, J. Med. Chem. 2014, 57, 1025710274.

[82] S. Herold, D. Bafaluy, K. Muñiz, Green Chem. 2018, 20, 3191-3196.

[83] P. Qian, Z. Yan, Z. Zhou, K. Hu, J. Wang, Z. Li, Z. Zha, Z. Wang, Org. Lett. 2018, 20, 6359-6363.

[84] K. Liu, C. Song, A. Lei, Org. Biomol. Chem. 2018, 16, 2375-2387.

[85] S. Zhang, L. Li, M. Xue, R. Zhang, Kun Xu, C. Zeng, Org. Lett. 2018, 20, 3443-3446.

[86] R. Sutcliffe, D. Griller, J. Lessard, K. U. Ingold, J. Am. Chem. Soc. 1981, 103, 624-628.

[87] X. Hu, G. Zhang, F. Bu, L. Nie, A. Lei, ACS Catal. 2018, 8, 9370-9375.

[88] M. A. Kabeshov, B. Musio, S. V. Ley, React. Chem. Eng. 2017, 2, 822-825.

[89] L. Eberson, K. Nyberg, Tetrahedron Lett. 1966, 22 , 2389-2393.

[90] N. Sbei, G. M. Martins, B. Shirinfar, N. Ahmed, Chem. Rev. 2020, 20, 1-24

[91] Y. Yuan, J. Qiao, Y. Cao, J. Tang, M. Wang, G. Ke, Y Lu, X. Liua, A. Lei, Chem. Commun. 2019, 55, 42304233.

[92] M. Huang, J. Dai, X. Cheng, M. Ding, Org. Lett. 2019 , 21, 7759-7762.

[93] A. Ollivier, S. Sengmany, M. Rey, T. Martens, E. Léonel, Synlett 2020, 31, 1191-1196.

[94] T. Raju, K. Kulangiappar, M. Anbu Kulandainathan, A. Muthukumaran, Tetrahedron Lett. 2005, 46, 70477050.

[95] M. Rafiee, F. Wang, D. P. Hruszkewycz, S. S. Stahl, J. Am. Chem. Soc. 2018, 140, 22-25.

[96] a) L. M. Reid, T. Li, Y. Cao, C. P. Berlinguette, $J$. Sustainable Energy Fuels 2018, 2, 1905-1927; b) J. P. Barham, B. König, Angew. Chem. Int. Ed. 2020, 59, $11732-11747$; c) Y. Wu, R. Song, J. Li, Org. Chem. Front. 2020, 7, 1895-1902; d) T. Hardwick, A. Qurashi, B. Shirinfar, N. Ahmed, ChemSusChem 2020, 13, 1967-1973; e) Y. Yu, P. Guo, J. Zhong, Y. Yuan, K. Ye, Org. Chem. Front. 2020, 7, 131-135.

[97] A. E. Becquerel, C. R. Acad. Sci. 1839, 9, 145-149.

[98] M. Grätzel, Nature 2001, 414, 338-344. 
[99] M. G. Walter, E. L. Warren, J. R. McKone, S. W. Boettcher, Q. Mi, E. A. Santori, N. S. Lewis, Chem. Rev. 2010, 110, 6446-6473.

[100] H. G. Cha, K. Choi, Nat. Chem. 2015, 7, 328-333.

[101] T. Li, T. Kasahara, J. He, K. E. Dettelbach, G. M. Sammis, C. P. Berlinguette, Nat. Commun. 2017, 8, 390.

[102] J.-H. Wang, X.-B. Li, J. Li, Tao Lei, H.-L. Wu, X.-L. Nan, C.-H. Tung, L.-Z. Wu, Chem. Commun. 2019, 55, 10376-10379.
[103] Y. Tang, Bo. Wu, H. He, C. Fu, J. Wang, K. Liu, G. Ke, Y. Zhou, J. Electrochem. Soc. 2019, 166, H662-H668.

[104] F. Wang, S. S. Stahl, Angew. Chem. Int. Ed. 2019, 58, 6385-6390.

[105] P. Xu, P. Chen, H. Xu, Angew. Chem. Int. Ed. 2020, 59, 14275-14280.

[106] L. Niu, C. Jiang, Y. Liang, D. Liu, F. Bu, R. Shi, H. Chen, A. D. Chowdhury, A. Lei, J. Am. Chem. Soc. 2020, 142, 17693-17702. 


\section{REVIEWS}

Photochemical and Electrochemical Strategies towards

Benzylic C-H Functionalization: A Recent Update

Adv. Synth. Catal. 2021, 363, 1-26

M. Oliva, G. A. Coppola, E. V. Van der Eycken, U. K. VIP Sharma*

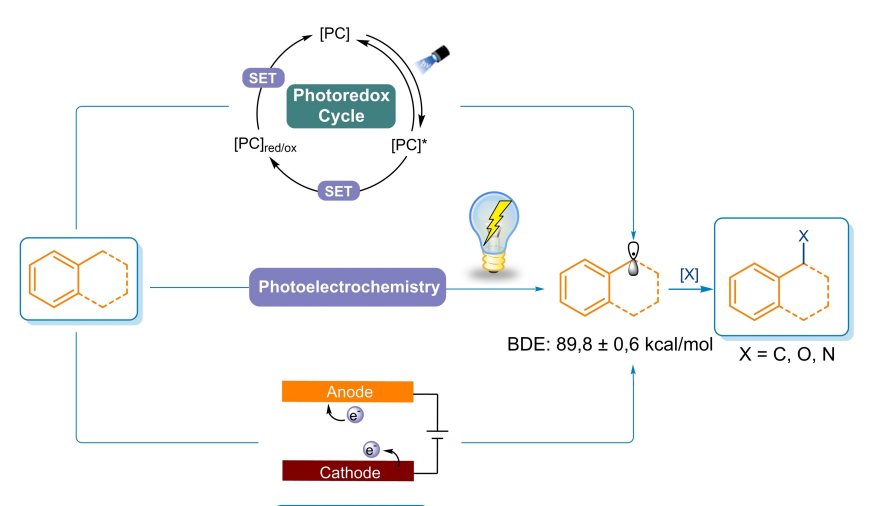

Electrochemistry 